\title{
Perspectives on Quenching and Tempering 4340 Steel
}

\author{
A.J. CLARKE, J. KLEMM-TOOLE, K.D. CLARKE, D.R. COUGHLIN, D.T. PIERCE, \\ V.K. EUSER, J.D. POPLAWSKY, B. CLAUSEN, D. BROWN, J. ALMER, P.J. GIBBS, \\ D.J. ALEXANDER, R.D. FIELD, D.L. WILLIAMSON, J.G. SPEER, and G. KRAUSS
}

\begin{abstract}
Steels are ubiquitous due to their affordability and the landscape of useful properties that can be generated for engineering applications. But to further expand the performance envelope, one must be able to understand and control microstructure development by alloying and processing. Here we use multiscale, advanced characterization to better understand the structural and chemical evolution of AISI 4340 steel after quenching and tempering (Q\&T), including the role of quench rate and short-time, isothermal tempering below $573 \mathrm{~K}\left(300{ }^{\circ} \mathrm{C}\right)$, with an emphasis on carbide formation. We compare the microstructure and/or property changes produced by conventional tempering to those produced by higher temperature, short-time "rapid" tempering. We underscore that no single characterization technique can fully capture the subtle microstructure changes like carbon redistribution, transition carbide and/or cementite formation, and retained austenite decomposition that occur during Q\&T. Only the use of multiple techniques begins to unravel these complexities. After controlled fast or slow quenching, $\eta$ transition carbides clearly exist in the microstructure, likely associated with autotempering of this high martensite start temperature $\left(M_{\mathrm{s}}\right)$ steel. Isothermal tempering below $598 \mathrm{~K}\left(325^{\circ} \mathrm{C}\right)$ results in the relief of carbon supersaturation in the martensite, primarily by the formation of $\eta$ transition carbides that exhibit a range of carbon levels, seemingly without substitutional element partitioning between the carbide and matrix phases. Hägg transition carbide is present between $300{ }^{\circ} \mathrm{C}$ and $325{ }^{\circ} \mathrm{C}$. After conventional tempering at or above $598 \mathrm{~K}$ $\left(325^{\circ} \mathrm{C}\right)$ for $2 \mathrm{~h}$, cementite is predominant, but small amounts of cementite are also present in other conditions, even after quenching. Previous work has indicated that silicon ( $\mathrm{Si}$ ) and substitutional elements partition between the cementite, which initially forms under paraequilibrium conditions, and the matrix. Phosphorous (P) may also be preferentially located at cementite/matrix interfaces after high temperature tempering. Slower quench rates result in greater amounts of retained austenite compared to those after fast quenching, which we attribute to increased austenite stability resulting from "autopartitioning". Rapid, high temperature tempering is also found to diminish tempered martensite embrittlement (TME) believed to be associated with the extent of austenite decomposition, resulting in mechanical properties not attainable by conventional tempering, which may have important implications with respect to industrial heat treatment processes like induction tempering. Controlling the amount and stability of retained austenite is not only relevant to the properties of Q\&T steels, but also next-generation advanced high strength steels (AHSS) with austenite/martensite mixtures.
\end{abstract}

https://doi.org/10.1007/s11661-020-05972-1

(c) The Minerals, Metals \& Materials Society and ASM International 2020

A.J. CLARKE, K.D. CLARKE, R.D. FIELD, and V.K. EUSER are with the Colorado School of Mines, 1500 Illinois St., Golden, CO 80401 and also with the Los Alamos National Laboratory, P.O. Box 1663, Los Alamos, NM 87545. Contact e-mail: amyclarke@mines.edu J. KLEMM-TOOLE, D.L. WILLIAMSON, J.G. SPEER, and G. KRAUSS are with the Colorado School of Mines. D.R. COUGHLIN, B. CLAUSEN, D. BROWN, P.J. GIBBS, and D.J. ALEXANDER are with the Los Alamos National Laboratory. D.T. PIERCE is with the Colorado School of Mines and also with the Oak Ridge National Laboratory, 1 Bethel Valley Rd., Oak Ridge, TN 37831. J.D. POPLAWSKY is with the Oak Ridge National Laboratory. J. ALMER is with the Advanced Photon Source, Argonne National Laboratory, 9700 Cass Ave., Lemont, IL 60439.

Manuscript submitted April 22, 2020.

Article published online August 27, 2020 


\section{INTRODUCTION}

A wide range of useful mechanical properties can be achieved by applying various tempering treatments to martensite in medium carbon, low alloy steels (Figure 1(a)). ${ }^{[1]}$ On the lower end of tempering temperatures typically employed by industry, often referred to as low temperature tempering (LTT), yield strengths well over $1 \mathrm{GPa}$, combined with tensile ductility exceeding 10 pct. culminating in ductile fracture, can be achieved. ${ }^{[2,3]}$ Determining the mechanisms responsible for ultrahigh strength, combined with appreciable ductility, requires a detailed understanding of the microstructural development during LTT. Changes to the as-quenched martensite microstructure have been classically divided into stages, based upon temperature ranges for a 1 hour tempering treatment. ${ }^{[4,5]}$ Below $100{ }^{\circ} \mathrm{C}$, interstitially dissolved carbon atoms can segregate to defects such as dislocations and boundaries. In the $100{ }^{\circ} \mathrm{C}$ to $250{ }^{\circ} \mathrm{C}$ range, transition carbides precipitate from carbon supersaturated martensite, which marks the first stage of tempering. The second stage of tempering involves the decomposition of retained austenite into ferrite and cementite, which occurs from $200{ }^{\circ} \mathrm{C}$ to $300{ }^{\circ} \mathrm{C}$. In the third stage of tempering, occurring in the range of $250{ }^{\circ} \mathrm{C}$ to $350{ }^{\circ} \mathrm{C}$, a microstructure consisting of cementite and ferrite is formed. ${ }^{[6,7]}$

For most practical applications, LTT is performed in the $150{ }^{\circ} \mathrm{C}$ to $200{ }^{\circ} \mathrm{C}$ range to maximize strength and ductility combinations, whereas tempering between $200{ }^{\circ} \mathrm{C}$ and $400{ }^{\circ} \mathrm{C}$ may lead to significant embrittlement. ${ }^{[1]}$ The primary microstructural changes concomitant with typical LTT treatments are transition carbide precipitation and the early stages of retained austenite decomposition. Significant embrittlement associated with tempering in the $200{ }^{\circ} \mathrm{C}$ to $400{ }^{\circ} \mathrm{C}$ range, termed tempered martensite embrittlement (TME) and typically reflected by a "trough" in the toughness vs. tempering

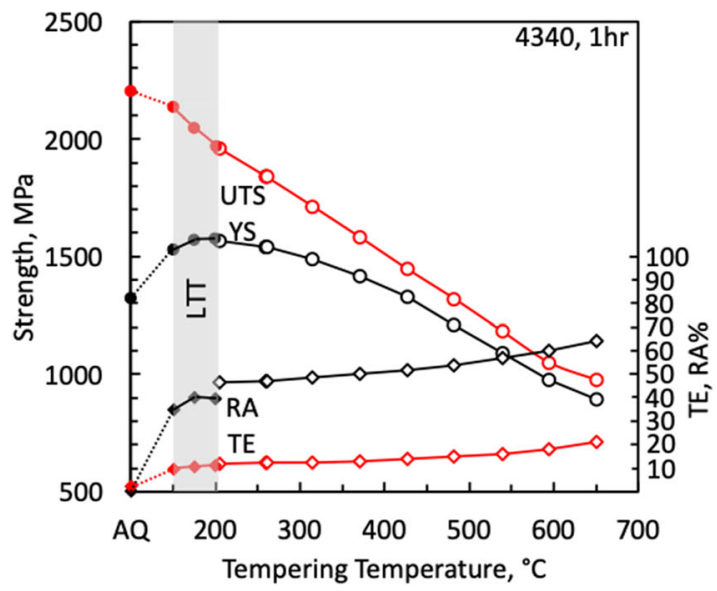

(a) curve, is associated with the formation of intra-lath cementite from retained austenite (Figure 1(b)). ${ }^{[8-12]}$ Furthermore, embrittlement caused by the segregation of elements such as phosphorus $(\mathrm{P})$ to cementite or prior austenite grain boundaries, termed temper embrittlement, may also occur after quenching from higher tempering temperatures. ${ }^{[8,11-14]}$ Accordingly, an in-depth understanding of the precipitation sequences and elemental segregation during tempering is of significant technological importance for the production of high strength, high toughness steels.

Numerous characterization techniques have been utilized to study microstructural evolution during tempering of medium carbon steel martensite. Dilatometry, or the measurement of volume changes associated with composition and crystal structure changes, has been effectively employed to identify all stages of tempering. ${ }^{[15-19]}$ Transmission electron microscopy (TEM) and electron diffraction have also been shown to be crucial for characterizing the morphology and crystal structure of transition carbides and cementite. Through careful microscopy and diffraction, Hirotsu and Nagakura showed that the transition carbide that forms in medium and high carbon steels during the first stage of tempering has an orthorhombic crystal structure, called $\eta$ carbide. ${ }^{[20,21]}$ Although there is some ambiguity in the distinction between the hexagonal transition carbide, $\varepsilon$, and the orthorhombic transition carbide $\eta,{ }^{[22,23]}$ several authors have suggested that diffraction patterns obtained after tempering are more consistent with $\eta$ than $\varepsilon .{ }^{[14,23-25]}$ Hägg or $\chi$ carbide has also been identified using TEM and diffraction to form as $\eta$ disappears and cementite forms in later stages of tempering. ${ }^{[26,27]}$ Mössbauer effect spectroscopy (MES) has been used as a valuable complement to TEM for the investigation of transition carbide formation during tempering. ${ }^{[7,23,26]}$ High-energy X-ray diffraction (HEXRD) from synchrotron sources has also been used with success to identify transition carbides, ${ }^{[15,28,29]}$ although the small

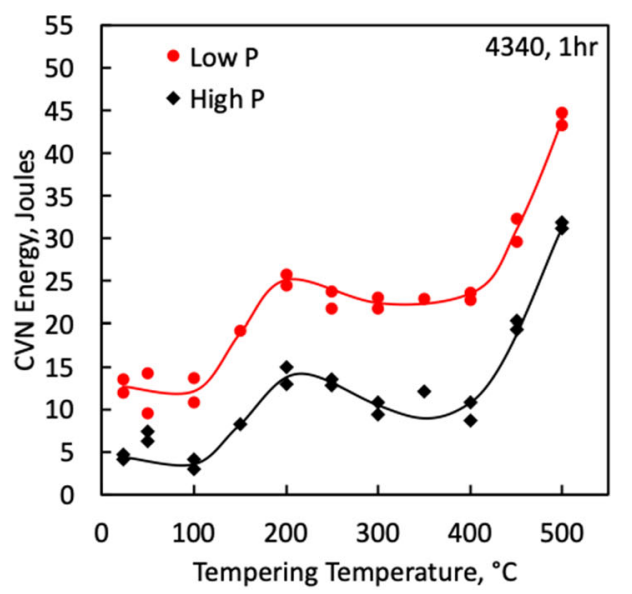

(b)

Fig. 1-(a) Example range of useful mechanical properties that can be produced in medium carbon, low alloyed 4340 steel by quenching and tempering (Q\&T). Replotted from Ref. [3]. (b) Impact (CVN) energy of 0.003 wt pct (Low P) and 0.03 wt pct (High P) 4340 steels, highlighting the tempered martensite embrittlement (TME) regime "trough", ranging from approximately $200{ }^{\circ} \mathrm{C}$ to $400{ }^{\circ} \mathrm{C}$. Replotted from Ref. [8] (Color figure online). 
sizes and volume fractions may cause problems with identification. ${ }^{[30-32]}$ To study highly localized composition changes during tempering, atom probe tomography (APT) has been extensively applied to evaluate tempering treatments in excess of $300{ }^{\circ} \mathrm{C}$, where substitution element mobility is sufficient to observe significant partitioning between ferrite/martensite and carbides. ${ }^{[14,33-40]}$ Authors have also used APT at tempering temperatures below $300{ }^{\circ} \mathrm{C}$, albeit to a lesser extent, to evaluate carbon and the early stages of substitutional element partitioning. ${ }^{[24,41-43]}$

In this study, we combine dilatometry, TEM, MES, HEXRD, and APT to develop a comprehensive understanding of the structural and composition changes that occur during quenching and tempering (Q\&T), with a focus on carbide formation at temperatures below $300{ }^{\circ} \mathrm{C}$ and quench rate effects on austenite amount, in the medium carbon (C), low alloy steel AISI 4340. These results are compared to microstructures and/or properties produced by tempering above $325^{\circ} \mathrm{C}^{[14]}$ and shorttime, "rapid" higher temperature tempering treatments of 4340 steel. $^{[44,45]}$ Implications with respect to industrial heat treatments and advanced high strength steels (AHSS) are also discussed.

\section{EXPERIMENTAL METHODS}

\section{A. Steel and Heat Treatments}

A medium C, 4340 low alloy steel was used for this study. The composition is provided in Table I. Nominally $6.25 \mathrm{~mm}$ thick plate was austenitized under vacuum for 30 minute at $845^{\circ} \mathrm{C}$ and oil quenched. Tempering was performed in air for 1 hour at $100^{\circ} \mathrm{C}$, $150{ }^{\circ} \mathrm{C}, 200{ }^{\circ} \mathrm{C}, 250{ }^{\circ} \mathrm{C}$, or $300^{\circ} \mathrm{C}$, or for 2 hour at $325^{\circ} \mathrm{C}$ or $450{ }^{\circ} \mathrm{C}$. Tempering for 2 hour at $575^{\circ} \mathrm{C}$ was performed under vacuum. Note that only results from selected Q\&T heat treatments are presented here.

Vacuum heat treatments were also performed using a high-rate induction dilatometer with gas-quenching capability. Solid $3 \mathrm{~mm}$ diameter and $10 \mathrm{~mm}$ long cylindrical samples with flat and parallel ends were heated between quartz platens. Length change was measured by a linear variable differential transformer (LVDT). All samples were heated to $850{ }^{\circ} \mathrm{C}$ in 60 second and held for 600 second to austenitize. Quench rate was varied by controlling He-gas flow to produce a "fast", and "slow" quench rate, resulting in cooling from $800{ }^{\circ} \mathrm{C}$ to 500 at rates of 167 and $25^{\circ} \mathrm{C} / \mathrm{s}$, respectively, with average cooling rates from the austenitizing temperature to $50{ }^{\circ} \mathrm{C}$ of 60 and $8{ }^{\circ} \mathrm{C} / \mathrm{s}$, respectively. Samples were held at room temperature $\left(25^{\circ} \mathrm{C}\right)$ for 200 second before heating at $10^{\circ} \mathrm{C} / \mathrm{s}$ to tempering temperatures of $100{ }^{\circ} \mathrm{C}, 200{ }^{\circ} \mathrm{C}$ or $300{ }^{\circ} \mathrm{C}$ for 100 or 300 second. All samples were quenched at approximately $20^{\circ} \mathrm{C} / \mathrm{s}$ from the tempering temperature. Temperatures were measured by a fine type-S thermocouple spot-welded to the mid-length surface of each sample.

\section{B. Atom Probe Tomography (APT)}

APT was performed using a local electrode atom probe (Cameca Instruments Inc. LEAP ${ }^{\circledR}$ 4000X HR). Sections were cut from Q\&T samples and mechanically ground to a thickness of approximately $0.25 \mathrm{~mm}$. Abrasive wire-cutting, followed by mechanical grinding, was used to fabricate specimen blanks with a square cross-section, nominally $0.25 \times 0.25 \mathrm{~mm}$. Specimen blanks were electropolished into sharp needles by a 2-stage double layer and micropolishing technique. ${ }^{[46]}$ The electrolytes used were 25 pct perchloric acid (70 pct perchloric acid, 30 pct water) in glacial acetic acid for rough polishing and 2 pet perchloric acid in 2-butoxyethanol for final polishing. ${ }^{[4]}$ Focused ion beam (FIB) annular milling was then performed in a FEI Nova 200 scanning electron microscope to sharpen each needle for APT. For the LEAP analyses, the specimen temperature was $50 \mathrm{~K}$ and the specimens were run in voltage mode with a pulse fraction of 0.2 and pulse repetition rate of $200 \mathrm{kHz}$. It is important to note that the compositional measurements from the APT data are influenced by the size of the studied feature, in which the accuracy of the compositional measurement decreases as the feature size decreases, due to local magnification effects (i.e., compositional information from the precipitates and neighboring matrix can artificially mix in the APT data). ${ }^{[47]}$ Additionally, compositional measurements of carbides can be influenced by the preferential loss of $\mathrm{C}$ or $\mathrm{Fe}$ containing ions during an atom probe measurement. ${ }^{[48]}$ Given the variable measured $\mathrm{C}$ levels of the carbides in this study, we note that APT alone should not be used to confidently identify carbides. We have made assumptions regarding the types of carbide captured in the APT datasets using the size and shape of the carbides, partitioning element behavior, and results from other techniques (such as TEM) from the same samples. Correlative APT with other techniques is needed to confidently identify the carbide types in APT data. ${ }^{[49]}$

\section{Transmission Electron Microscopy}

Transmission electron microscopy (TEM) specimens were prepared by electropolishing or FIB milling. For the electropolished samples, standard $3 \mathrm{~mm}$ TEM discs were machined from heat-treated samples, including samples heat-treated by dilatometry, and electropolished using a Struers twin jet electropolisher using a 95

Table I. 4340 Steel Composition

\begin{tabular}{lccccccrrr}
\hline & $\mathrm{C}$ & $\mathrm{Mn}$ & $\mathrm{Si}$ & $\mathrm{Ni}$ & $\mathrm{Cr}$ & $\mathrm{Mo}$ & $\mathrm{Cu}$ & $\mathrm{P}$ & $\mathrm{S}$ \\
\hline Wt Pct & 0.42 & 0.78 & 0.26 & 1.78 & 0.83 & 0.24 & 0.03 & 0.010 \\
At. Pct & 1.92 & 0.78 & 0.51 & 1.67 & 0.88 & 0.14 & 0.03 & 0.018 & 0.003 \\
\hline
\end{tabular}


pct glacial acetic acid with 5 pet perchloric acid solution. The FIB foil preparation was performed in a FEI DB235 dual-beam FIB equipped with an Omniprobe nano-manipulator for foil extraction. TEM foils were analyzed with a FEI Tecnai F30 operated at $300 \mathrm{kV}$. The microscope was operated in conventional TEM mode to acquire bright field (BF) and dark field (DF) images and selected area diffraction patterns (SADPs). The focus of the TEM analysis was to characterize and identify the carbides present in the microstructure. SADPs were also simulated with Desktop Microscopist software to confirm the correct indexing of g-vectors. The following orientation relationship was used for different variants of $\eta$ transition carbide ${ }^{[20,21]}$ :

$$
\begin{array}{r}
(110)_{\eta 1} \|(100)_{\alpha} \\
{[001]_{\eta 1} \|[010]_{\alpha}} \\
(110)_{\eta 2} \|(010)_{\alpha} \\
{[001]_{\eta 2} \|[100]_{\alpha}}
\end{array}
$$

The Bagaryatsky orientation relationship was used for cementite $(\theta) \cdot{ }^{[50]}$

$$
\begin{aligned}
& (211)_{\alpha} \|(010)_{\theta} \\
& {[0 \overline{1} 1]_{\alpha} \|[001]_{\theta}}
\end{aligned}
$$

\section{High-Energy X-ray Diffraction (HEXRD)}

Samples were measured during two different beam time allocations at the Advanced Photon Source (APS) at Argonne National laboratory (ANL). Two slightly different instrument configurations were used, which are not expected to influence any of the refined phase fractions or the identification of carbides. Where different, the instrument parameters mentioned below are given separated by a "/" for the two setups. For this work, Sector 1-ID-E received a monochromated beam from an APS superconducting undulator and a double crystal Laue monochromator with an energy of $E=$ $65.015 / 61.333 \mathrm{keV}$ designed for high-energy X-rays. For diffraction experiments, the incident beam was masked to a $0.2 \times 0.2 / 0.1 \times 0.1 \mathrm{~mm}^{2}$ cross section. Scattered $\mathrm{x}$-rays were collected on a GE $41 \mathrm{RT}$ area detector with $2048 \times 2048$ pixels $\left(0.2 \times 0.2 \mathrm{~mm}^{2}\right.$ pixel size $)$ centered on the straight-through beam and positioned 1453.35/ $833.16 \mathrm{~mm}$ from the sample, covering roughly $\pm 14.7 /$ $13.2 \mathrm{deg}$ of $2 \theta$. Positioned as such, the angular coverage of the detector was sufficient to collect five or more complete Debye-Scherrer diffraction rings for both the austenite (face-centered cubic) and ferrite (body-centered cubic) phases of the steel. The detector was operated in multi-frame mode, collecting a total of 100 frames, each integrated between 0.5 to 2 second, depending on sample texture and thickness to avoid saturation. The 100 frames were then summed in the GSAS-II software ${ }^{[51]}$ to obtain better statistics on the small carbide peaks, and a single powder diffraction pattern was generated by integrating the diffraction images over the full 360 degrees. It was only possible to quantitatively refine the ferrite and austenite; identification of the carbides was visually estimated considering the locations of the most intense and visible peaks for each phase.

\section{E. Mössbauer Effect Spectroscopy (MES)}

Austenitized, quenched, and tempered samples were prepared for MES by gentle mechanical polishing to $100 \mu \mathrm{m}$, and then by chemical polishing below $30 \mu \mathrm{m}$ to remove the deformation layer. The chemical polishing solution was 10:10:1 de-ionized water: hydrogen peroxide (70 pct water, 30 pct $\mathrm{H}_{2} \mathrm{O}_{2}$ ): hydrofluoric acid (52 pct water, 48 pct HF). MES spectra were obtained at room temperature with a ${ }^{57} \mathrm{Co}-\mathrm{Rh}$ source until sufficient data were obtained for each sample. The data, comprising counts for each velocity, were normalized, and subspectra were fit using Lorentzian profiles with the WinNormos V3.0 coupled with the IGOR Pro V6.3 software package. A methodology has been developed for detecting and quantifying small amounts of carbides and austenite in steels. ${ }^{[52,53]}$ This requires collecting high statistical quality data and carefully fitting the dominant martensite/ferrite resonance with multiple magnetic sextets to account for the alloying effects, thereby revealing the weak contributions from the transition carbide and cementite phases. The carbides have significantly smaller magnetic hyperfine fields than the martensite/ferrite that lead to partial resolution of their subspectra. This is demonstrated in Figure 10 of Reference 52 and Figure 1 of Reference 53. Austenite is readily detected, since it is non-magnetic and therefore well-resolved in the central velocity region of the spectrum. Resonance area detection limits of about 0.3 and $0.1 \mathrm{pct}$ for carbides and austenite, respectively, have been established. Accurate quantification is done by making thickness corrections and accounting for differences in recoilless fractions for the various phases, as described in detail in References 52 and 53. This MES methodology has been applied recently to other steel research. ${ }^{[16,44,58]}$

\section{RESULTS AND DISCUSSION}

\section{A. Dilation Response During $Q \& T$}

"Fast" and "slow" quench rates were used for the dilatometry heat treatments to make two sets of Q\&T microstructures for direct comparison. Temperature-time profiles are shown in Figure 2(a). Offset strain change ( $\Delta$ Strain) as a function of temperature is shown for the slow (red) and fast (blue) quench sets in Figure 2(b). The fast quench set exhibits less consistent dilation behavior at higher temperatures than the slow quench set, which is likely associated with larger temperature gradients within the sample. The fast quench set also displays apparent variations in martensite start $\left(M_{\mathrm{s}}\right)$ temperature compared to the slow quench set, which may also be associated with larger 
temperature gradients in the fast quench samples. Significant strain changes do not occur during tempering below $300{ }^{\circ} \mathrm{C}$ for 100 or 300 second (Figure 2(c)), but at $300{ }^{\circ} \mathrm{C}$, volume contraction occurs at short times, indicating measurable microstructural changes associated with martensite tempering.

\section{B. Microstructure After Fast and Slow Dilatometric Quenching}

TEM was first used to characterize the fast and slow quenched microstructures in the as-quenched condition (without tempering). Figure 3(a) is an off-zone axis BF TEM image, revealing a martensitic microstructure that contains plate-like carbides within martensite laths produced by fast quenching. The SADPs shown in Figures $3(\mathrm{~b})$ and (c) are from the $[11 \overline{1}]_{\alpha}$ and $[31 \overline{1}]_{\alpha}$ zone axes, respectively, and were taken from the same area shown in Figure 3(a). Figure 3(d) shows an off-zone axis $\mathrm{BF}$ image of martensite after slow quenching with SADPs (Figures 3(e) and (f)) from the $[\overline{100}]_{\alpha}$ and $[\overline{101}]_{\alpha}$ zone axes. It should be noted that every TEM sample was assumed to contain surface oxide in the form of magnetite. Magnetite reflections are observable in some of the SADPs, but in general the intensities of the reflections were too low to be seen. Additionally, magnetite reflections can overlap with $\eta$ transition carbide reflections in some instances, as noted in subsequent figure captions. The predominant carbide is $\eta$ transition carbide in both as-quenched conditions, consistent with earlier TEM observations, ${ }^{[14]}$ suggesting autotempering of this high $M_{\mathrm{s}}$ temperature (Figure 2) steel occurs during quenching. In Reference 14, in addition to $\eta$ transition carbide identified by TEM, ultrafine scale carbon clustering, signifying potential carbon redistribution to defects and boundaries in the martensite, has also been revealed by APT. Although not shown here, similar carbon clustering was also revealed after fast and slow dilatometric quenching, in addition to $\eta$ transition carbide. The presence of $\eta$ transition carbide, and thus autotempering, has been reported in other medium carbon steels following oil quenching using MES. ${ }^{[16]}$ Although $\eta$ transition carbide has been identified, it remains challenging to quantify differences between the fast and slow quenched conditions in this work. Detailed MES measurement would help in this regard. That said, quench rate is found to play an important role on other aspects of microstructural evolution, such as the amount of retained austenite, as discussed later.

HEXRD of selected conditions was performed at the APS at ANL. Figure 4 compares the results obtained after fast and slow dilatometric quenching, highlighting selected peak positions of relevant phases surrounding a high intensity ferrite peak in the center of each diffractogram. The stick markings in Figure 4 help to indicate the presence of specific phases for clarity, although note that the locations of additional peaks corresponding to relevant phases are also provided in Figure 4 by tick marks. The existence of $\eta$ transition carbide is clearly observed in both conditions, in agreement with the TEM results shown in Figure 3. As noted previously, the unambiguous identification of $\eta$ from $\varepsilon$ transition carbide by TEM is difficult, ${ }^{[14,22-24]}$ but these complementary HEXRD results corroborate the identification of $\eta$ transition carbide by TEM in this work. It is also interesting to note that a small amount of cementite seems to be present in both conditions after austenitizing and quenching. The amount of austenite is also greater in the slow quenched condition, relative to the fast quench condition, based upon Rietveld refinement of ferrite and austenite; the qualitative difference in austenite amount is also revealed by the relative difference in the austenite peak intensities (Figure 4).

The aim of austenitizing prior to quenching is to form nominally homogenous austenite with the greatest possible amount of $\mathrm{C}$ and other alloying elements in solution to promote hardenability and the formation of martensite during quenching. That said, aspects (length scale, chemical homogeneity, etc.) of prior microstructure going into the austenitizing step may play an important role on subsequent microstructural evolution. In medium $\mathrm{C}$, low alloy steels, it is typically assumed that the austenite achieves compositional homogeneity

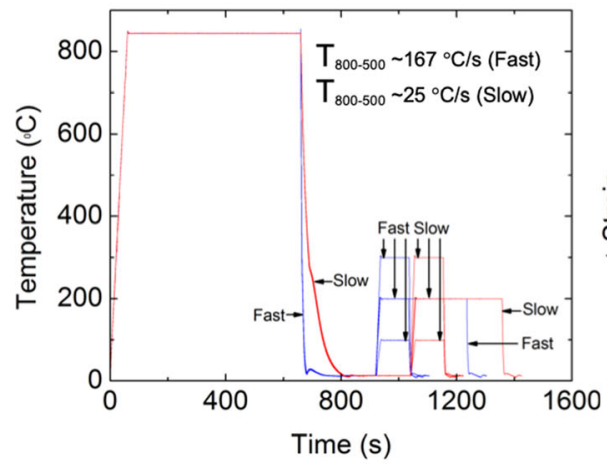

(a)

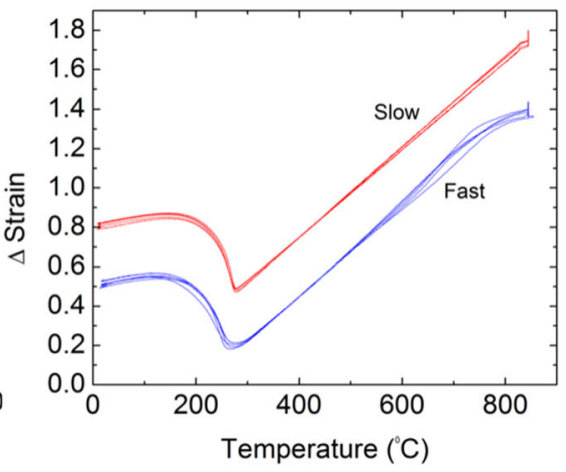

(b)

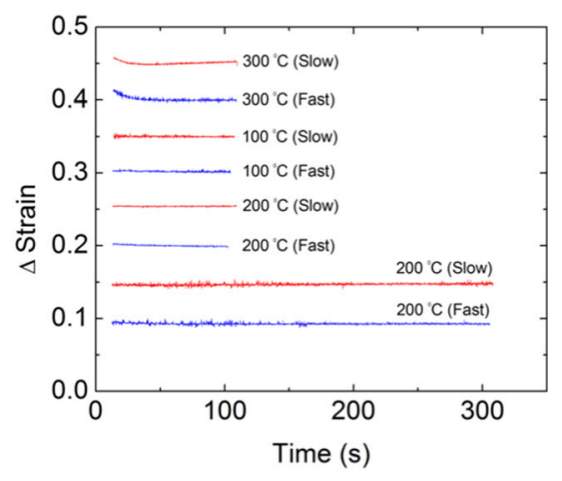

(c)

Fig. 2- Q\&T of 4340 steel: (a) temperature-time profiles obtained from dilatometry, $(b)$ offset strain change vs. temperature for slow and fast quenching, and $(c)$ offset strain changes during tempering for slow and fast quenching. The quench rate from $800{ }^{\circ} \mathrm{C}$ to $500{ }^{\circ} \mathrm{C}$ ( $\mathrm{T}_{800-500}$ ) is $\sim 167$ ${ }^{\circ} \mathrm{C} / \mathrm{s}$ for fast quenching and $\sim 25{ }^{\circ} \mathrm{C} / \mathrm{s}$ for slow quenching. Short-time tempering was performed at $100{ }^{\circ} \mathrm{C}, 200{ }^{\circ} \mathrm{C}$, or $300{ }^{\circ} \mathrm{C}$ for 100 or $300 \mathrm{~s}$. Reprinted from Ref. [54] (Color figure online). 

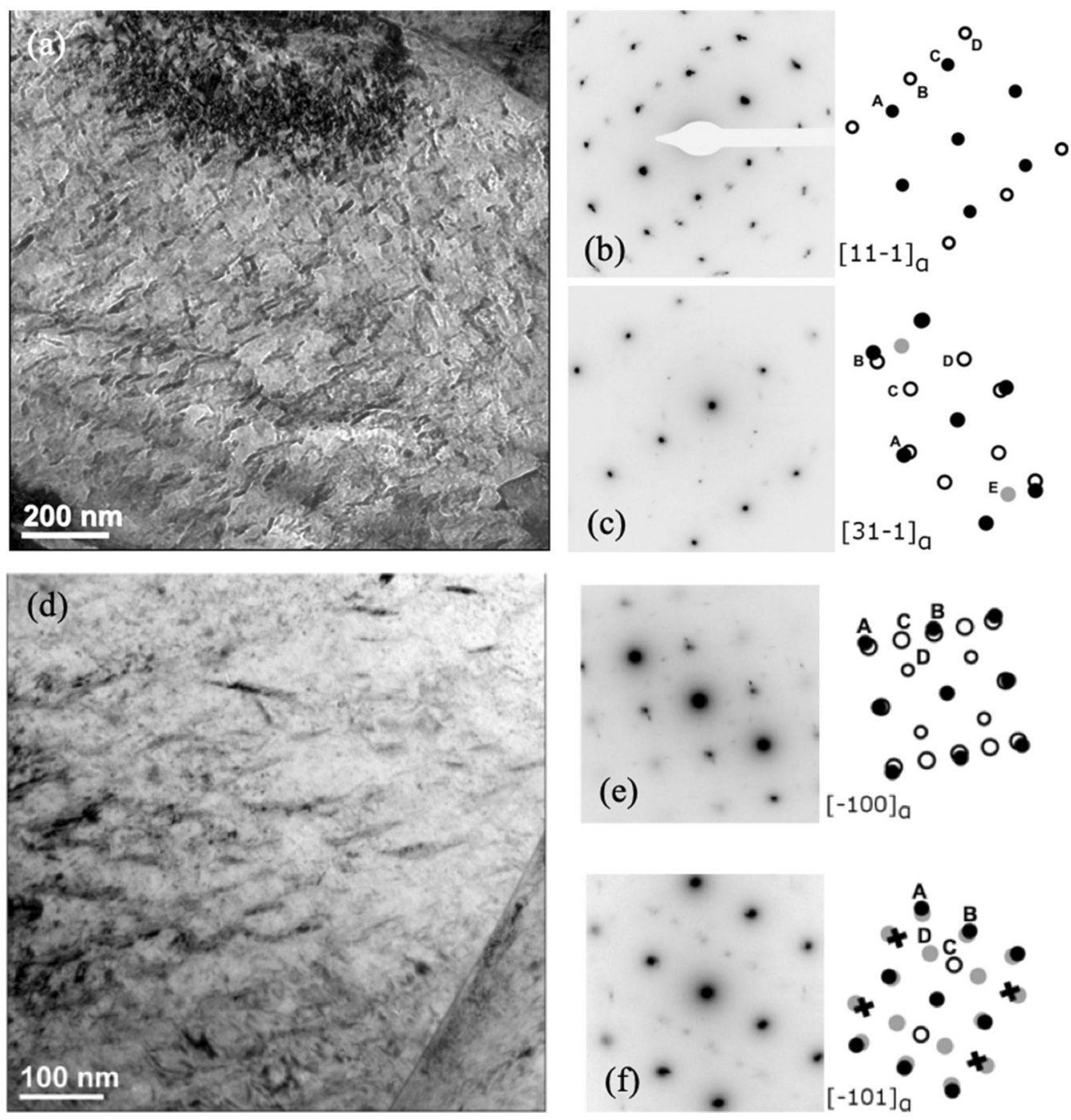

Fig. 3- (a) BF TEM image (off-zone) of $\eta$ transition carbides in 4340 steel after fast quenching in a dilatometer, (b) SADP of a [11 $\overline{1}]_{\alpha}$ zone axis; $\alpha=(011)_{\alpha}, B=(0 \overline{1} 1)_{,}, C=(\overline{1} 10)$, and $D=(\overline{2} \overline{1} 1)_{n},(c)$ SADP of a $[31 \overline{1}]_{\alpha}$ zone axis; $\alpha=(0 \overline{1} \overline{1}), B=(1 \overline{2} 1), C=(10 \overline{1})_{n}, D=(2 \overline{1} 0)$, (surface oxide, magnetite, can also contribute to the intensity shown at this g-vector), and $E=(30 \overline{1})_{\eta_{2}}$. (d) BF TEM image (off-zone) of ${ }^{\eta_{1}}$ transition carbides in 4340 steel after slow quenching in a dilatometer. (e) SADP of a $[\overline{1} 00]$ zone axis; $\alpha^{\eta_{2}}=(00 \overline{2}), B=(0 \overline{1} 1) a, C=(2 \overline{1} 0)$, and $D=(1 \overline{1} 0)_{\eta_{1}}$ (surface oxide, magnetite, can also contribute to the intensity shown at this g-vector). ( $f$ ) SADP of a $[\overline{101}]_{\alpha}$ zone axis; $X \stackrel{\eta_{1}}{=}$ $\{440\}_{O}$ (surface oxide, magnetite), $\alpha=(\overline{1} 2 \overline{1}), B=(020)_{\alpha}, C=(110)_{\eta_{1}}$ (surface oxide, magnetite, can also contribute to the intensity shown at this g-vector), and $D=(\overline{101})_{\eta_{2}}$ (note: all SADPs were taken from the areas shown in the corresponding BF TEM images).

during austenitization, but in many instances pre-existing microstructural features such as carbides may not fully dissolve. Even if dissolved, these features may leave remnant segregation of partitioned elements. ${ }^{[54-57]}$ Undissolved carbides from incomplete austenitization have been shown to significantly influence fracture properties after tempering. ${ }^{[3,12,13]}$ Microstructure before austenitizing may also contain mesoscopic chemical inhomogeneity, such as $\mathrm{Mn}$ banding, which austenitization may not eliminate. ${ }^{[55-57]}$ Regardless of the source, incomplete dissolution of prior microstructural constituents and/or element homogenization may result in retained features from the prior microstructure and/or local variations in chemical phase stability, which may then influence subsequent phase transformations, perhaps including carbide nucleation and/or growth.
Figure 5(a) shows an APT C map after slow dilatometric quenching, with 5 at. pct iso-concentration surfaces bounding $\mathrm{C}$-containing regions at or above this level. A proximity histogram is shown in Figure 5(b) of the highlighted (pink) region in Figure 5(a). Chromium (Cr), molybdenum (Mo), and manganese (Mn) are partitioned to this $\mathrm{C}$-enriched feature. In previous work, cementite was revealed by APT in the same 4340 steel after tempering at $325{ }^{\circ} \mathrm{C}$ for 2 hours or above. ${ }^{[14]}$ It was reported that early stage cementite growth initially occurs under paraequilibrium conditions, with eventual Si redistribution out of the cementite and into the matrix, followed by $\mathrm{Cr}, \mathrm{Mn}$, and Mo partitioning to the cementite, particularly near the cementite/matrix interfaces, at higher tempering temperatures. ${ }^{[14]}$ Given the extent of partitioning associated with the $\mathrm{C}$-enriched feature in Figure 5 and the HEXRD results provided in 


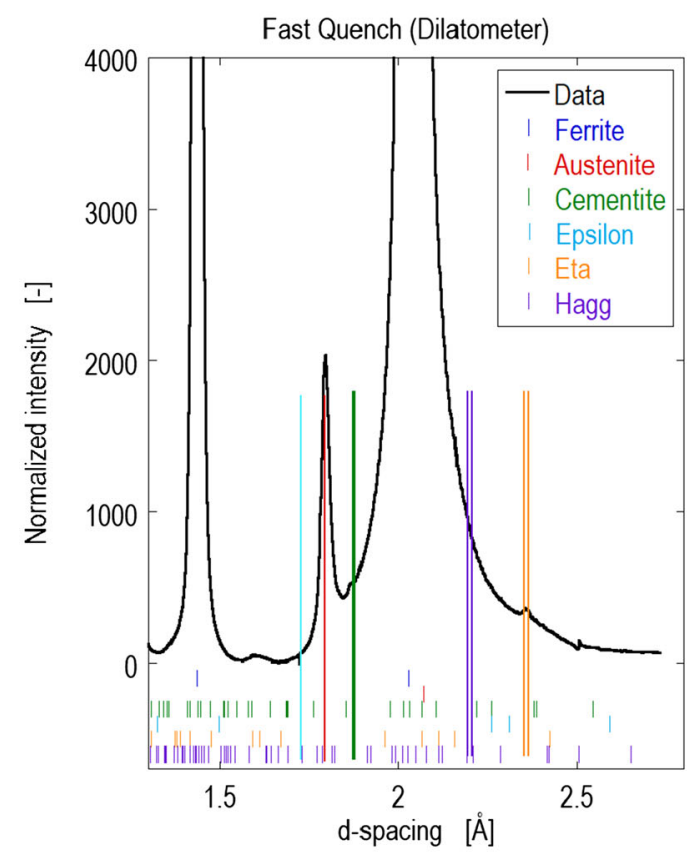

(a)

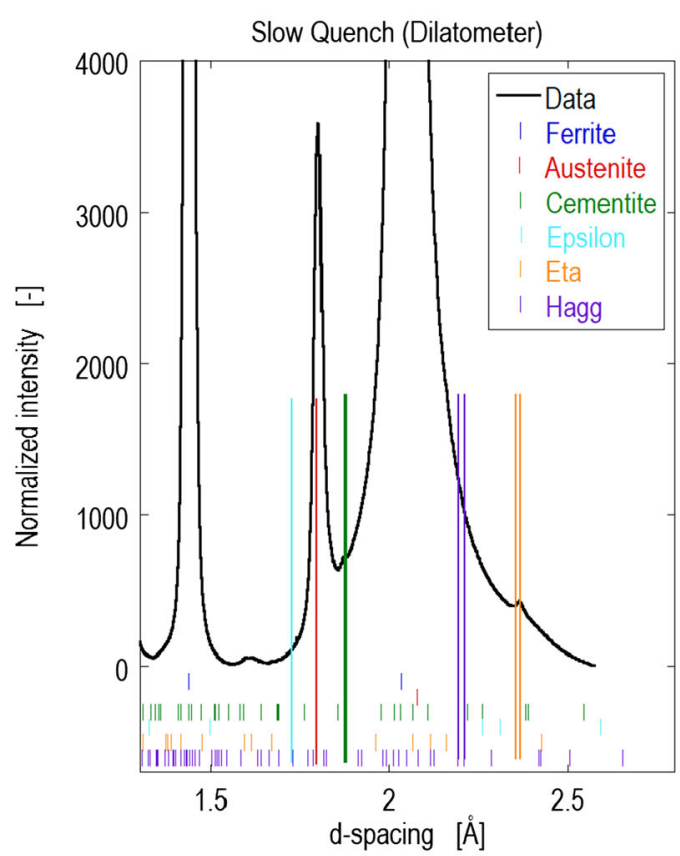

(b)

Fig. 4-HEXRD of 4340 steel after $(a)$ fast quenching and $(b)$ slow quenching in the dilatometer. Note the prescence of eta $(\eta)$ transition carbide and some cementite $(\theta)$, in addition to austenite $(\gamma)$ and ferrite $(\alpha)$. Tick marks highlight peak positions for the relevant phases, and the larger stick markings indicate peak positions of specific phases surrounding the high intensity ferrite peak to facilitate phase identification (Color figure online).

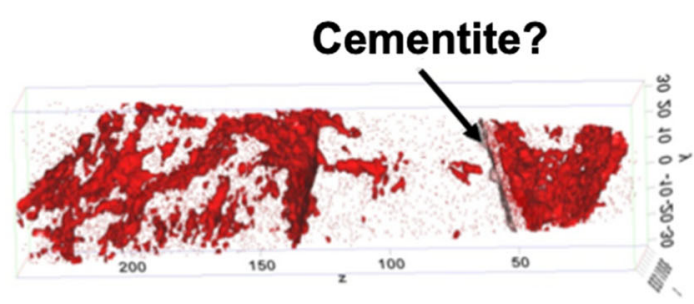

(a)

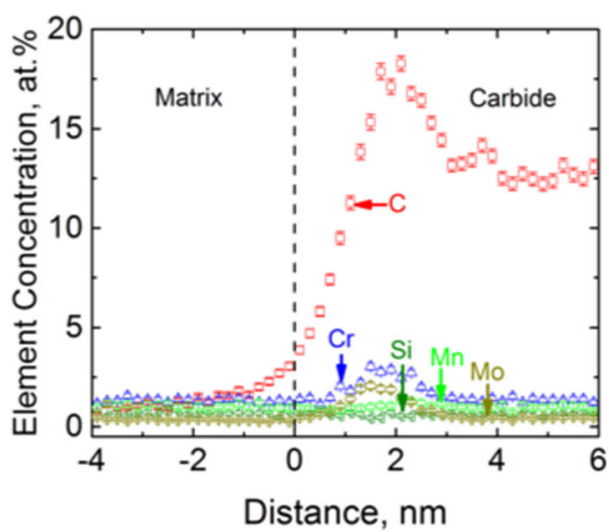

(b)

Fig. 5-APT of 4340 steel after slow quenching in the dilatometer. (a) APT C map and 5 at. pct C iso-concentration surfaces (scale in nms) and (b) a proximity histogram of the region highlighted in (a). Based upon the extent of observed element (Cr, Mo, Mn) partitioning, the highlighted region is thought to be cementite remaining after incomplete austenitization (Color figure online).

Figure 4 that suggest some cementite is present after quenching, it is speculated that the $\mathrm{C}$-enriched feature in Figure 5 with evolved element partitioning is cementite remaining in the microstructure after incomplete dissolution during austenitization before quenching. One would expect early stage cementite that potentially forms during autotempering of this high $M_{\mathrm{s}}$ temperature steel (and any $\eta$ transition carbide formed by autotempering (Figure 3), as presented later), to not have evolved element partitioning, given our previous study of tempering at or above $325^{\circ} \mathrm{C},{ }^{[14]}$ as significant substitutional partitioning with respect to the cementite/matrix was not observed until higher temperatures. The lack of element partitioning expected for early stage, paraequilibrium cementite makes it difficult to unambiguously distinguish it from $\eta$ transition carbide after quenching or tempering below $300{ }^{\circ} \mathrm{C}$ by composition alone, as discussed later, but the extent of partitioning observed in Figure 5 suggests "evolved" cementite, more like that seen after tempering above 
$325{ }^{\circ} \mathrm{C} \cdot{ }^{[14]}$ In addition to possible cementite remaining after austenitizing and $\eta$ transition carbide and cementite formation by autotempering, the possible formation of carbides in austenite must also be considered. A recent study of $\mathrm{Fe}-\mathrm{C}-\mathrm{Si}-\mathrm{Mn}-\mathrm{Nb}$ steels processed by quenching and partitioning (Q\&P) to create austenite/martensite mixtures revealed the presence of cemenite and $\chi$ (or Hägg) carbide by in-situ HEXRD, along with a $\chi$ carbide in the austenite after partitioning at $420{ }^{\circ} \mathrm{C},{ }^{[29]}$ perhaps stimulating the need for studies of carbide formation in austenite relative to the $M_{\mathrm{s}}$ temperature in the context of this work.

\section{Microstructure After $Q \& T$ at 100 and $200{ }^{\circ} \mathrm{C}$}

Following the example of evolved cementite possibly resulting from incomplete austenitization shown in Figure 5, APT data from part of a large, Cr-containing $\mathrm{M}_{23} \mathrm{C}_{6}$ carbide after austenitizing, oil quenching, and tempering at $200{ }^{\circ} \mathrm{C}$ for $1 \mathrm{~h}$ is shown in Figures 6(a) through (c). A large Cr-containing $\mathrm{M}_{23} \mathrm{C}_{6}$ carbide identified by TEM (Figures 6(d), through (f)) is also shown. TEM chemical analysis of the carbide shown in Figure 6(f) also revealed significant levels of $\mathrm{Cr}$ and $\mathrm{Mo}$ in the carbide, in agreement with the APT results shown in Figure 6(c). P segregation at the carbide/matrix interface is also observed by APT, which is known to degrade fracture properties via temper embrittlement. ${ }^{[8,11-13]}$ The existence of large,
$\mathrm{M}_{23} \mathrm{C}_{6}$ carbides in this condition may indicate incomplete carbide dissolution during austenitization, which has the potential to result in local microstructural and hardenability variations during subsequent $\mathrm{Q} \& \mathrm{~T}$. The presence of $\mathrm{M}_{23} \mathrm{C}_{6}$ carbides suggests that further control of upstream processing may be warranted.

Example APT results after fast dilatometric quenching, followed by short-time tempering at $100{ }^{\circ} \mathrm{C}$ or $200{ }^{\circ} \mathrm{C}$ for 100 second, are shown in Figures 7(a) and (b) and $8(\mathrm{a})$ and (b). These results are compared to conventional low temperature tempering for 1 hour at $100{ }^{\circ} \mathrm{C}$ or $200{ }^{\circ} \mathrm{C}$ in Figures 7(c) and (d) and 8(c) and (d), respectively. C-containing, plate-like features revealed by 5 at. pct iso-concentration surfaces are displayed. Proximity histograms of the highlighted features exhibit variable $\mathrm{C}$ levels, ranging up to approximately 20 at. pct, without substitutional element $(\mathrm{Cr}$, Mo, Mn) partitioning within the carbides and matrix. Additionally, no Si, Al, or Ni partitioning were observed by APT. TEM analysis after tempering at $100{ }^{\circ} \mathrm{C}$ or $200{ }^{\circ} \mathrm{C}$ revealed carbides like those shown in Figure 3 after fast or slow dilatometric quenching; $\eta$ transition carbides and cementite were also identified by HEXRD, similar to the results shown in Figure 4. APT results after fast and slow dilatometric quenching are also similar to those shown in Figures 7 and 8, which in addition to carbides also includes carbon redistribution to defects and boundaries in the martensite. ${ }^{[14,32]}$ Given (a)

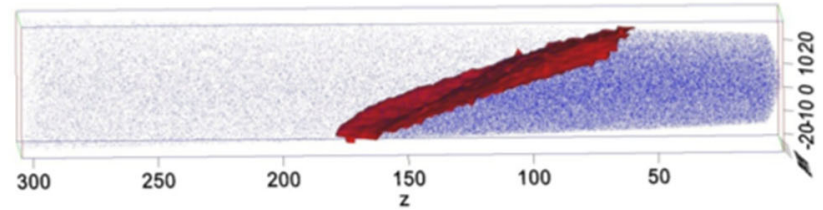

(b)
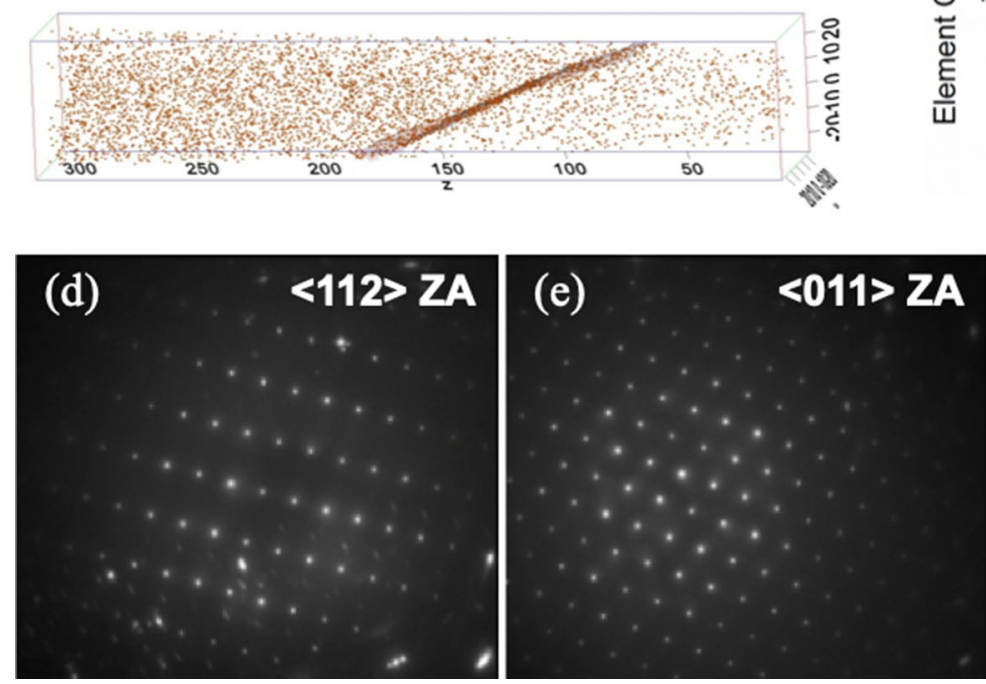
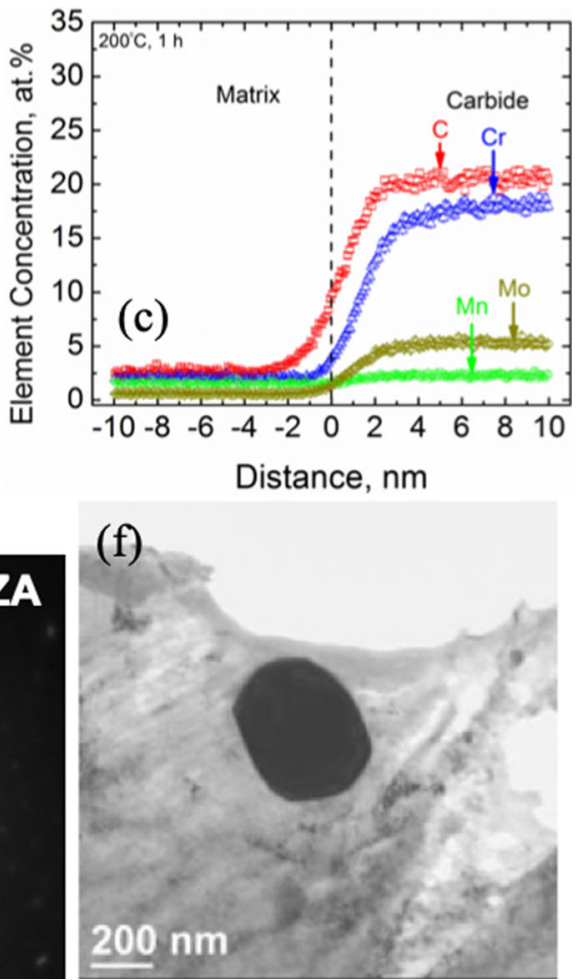

Fig. 6- (a) APT $\mathrm{Cr}$ map, highlighting $\mathrm{Cr}$ partitioning within an $\mathrm{M}_{23} \mathrm{C}_{6}$ carbide, and a 10 at. pct $\mathrm{C}$ iso-concentration surface after Q\&T at 200 ${ }^{\circ} \mathrm{C}$ for $1 \mathrm{~h},(b)$ an APT P map showing segregation at the carbide/matrix interface, $(c)$ a proximity histogram for the iso-concentration surface shown in (a), highlighting $\mathrm{Cr}$, Mo, and $\mathrm{Mn}$ partitioning within an $\mathrm{M}_{23} \mathrm{C}_{6}$ carbide. Reprinted from Ref. [54]. (d,e) TEM SADPs of $\langle 112\rangle$ and $\langle 011\rangle$ zones axes of a face-centered cubic (FCC) $(\mathrm{a} \sim 1.004 \mathrm{~nm}) \mathrm{Cr}$ - and Mo-containing $\mathrm{M}_{23} \mathrm{C}_{6}$ carbide (confirmed by elemental mapping not shown here) after Q\&T at $200{ }^{\circ} \mathrm{C}$ for $1 \mathrm{~h}$ of 4340 steel, and $(f)$ a BF TEM image of the $\mathrm{M}_{23} \mathrm{C}_{6}$ carbide in (d, e) (Color figure online). 


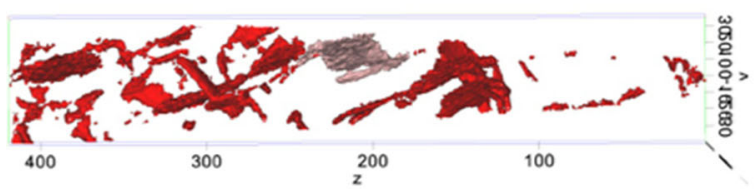

(a)

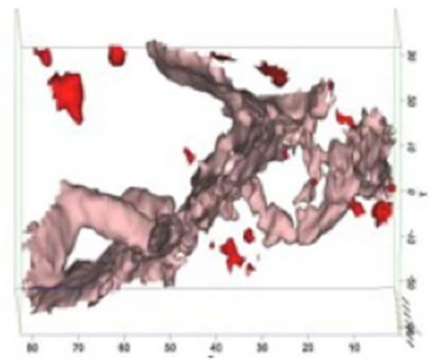

(c)

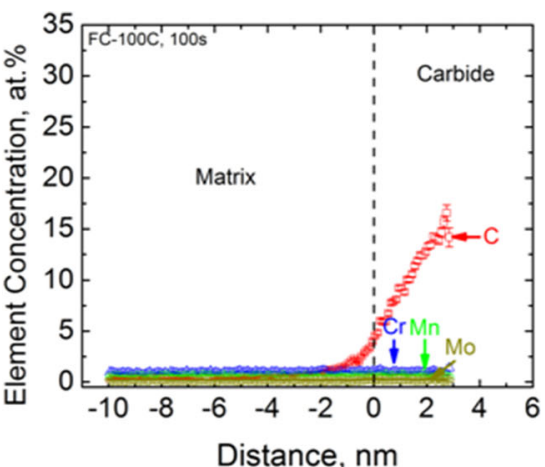

(b)

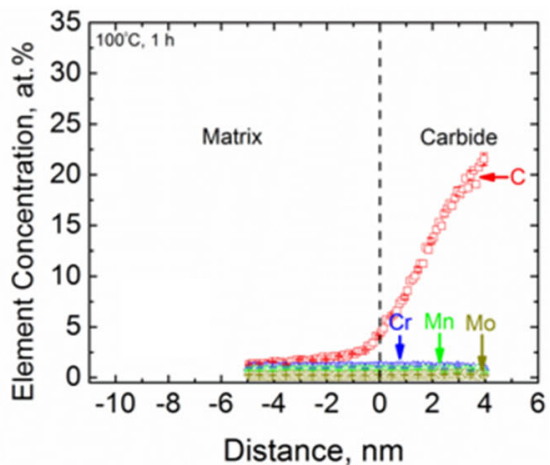

(d)

Fig. 7-(a) An APT C map (scale in nms) with 5 at. pct $\mathrm{C}$ iso-concentration surfaces of 4340 steel after fast quenching in the dilatometer and tempering at $100{ }^{\circ} \mathrm{C}$ for $100 \mathrm{~s},(b)$ a proximity histogram of the region highlighted in (a), (c) an APT C map (scale in nms) of 4340 steel with 5 at. pct $\mathrm{C}$ iso-concentration surfaces after oil quenching and tempering at $100{ }^{\circ} \mathrm{C}$ for $1 \mathrm{~h}$, and $(d)$ a proximity histogram of the region highlighted in (c) (Color figure online).

these results, it is believed that the carbides shown after LTT (e.g., Figure 7(a)) for both quench rates likely correspond to $\eta$ transition carbides, although as noted $\mathrm{C}$ segregation to defects and boundaries are also anticipated. That said, one would expect higher $\mathrm{C}$ levels (near $\sim 30$ at. pct) for $\eta$ transition carbide than those observed by APT. Lu et al. also reported lower than expected $\mathrm{C}$ levels in $\eta$ carbide formed in a Fe-15Ni-1C (wt pct) alloy quenched and tempered at room temperature for up to 3 years. ${ }^{[2]}$ In earlier work, it was previously speculated that non-stoichiometric $\eta$ transition carbide ${ }^{[20,52,54,58,59]}$ might also be present after LTT based upon TEM and APT results, but this remained to be unambiguously confirmed by another advanced characterization technique. ${ }^{[54]}$ Later we show that the majority of $\eta$ transition carbide is stoichiometric, as determined by MES, but some non-stoichiometric $\eta$ transition carbide also exists. We believe the low and variable carbon levels measured by APT are associated with challenges in measuring these fine-scale, C-containing features with APT, due to local magnification aberrations and other issues, ${ }^{447,48]}$ which is supported by other researchers. ${ }^{[2]}$ Given the variable $\mathrm{C}$ levels and lack of partitioning (also shown by early stage (paraequilibrium) cementite ${ }^{[14]}$ ), we note that APT alone should not be used to identify carbides. Supplemental information from complementary techniques is needed. That said, there is value in seeing the morphologies of the carbon-enriched features, which may help to determine if they correspond to carbides or
C segregation to defects and boundaries in the martensite. ${ }^{[14,32]}$

\section{Microstructure After $Q \& T$ at $250{ }^{\circ} \mathrm{C}$}

The off-zone BF TEM image (Figure 9(a)) after oil quenching and conventional tempering at $250{ }^{\circ} \mathrm{C}$ for 1 hour reveals $\eta$ transition carbides. SADPs from the $[100]_{\alpha}($ Figure $9(\mathrm{~b}))$ and $[31 \overline{1}]_{\alpha}$ (Figure 9(c)) zone axes, along with SADP schematics from the same area as the BF TEM image in Figure 9(a) are also shown. APT results for the same condition are also shown in Figure 9(d) through (g). The 5 at. pct $\mathrm{C}$ iso-concentration surfaces reveal plate-like features, whereas the proximity histograms of the highlighted features exhibit variable $\mathrm{C}$ levels and no substitutional element partitioning, similar to the results shown in Figures 7 and 8, suggesting the presence of $\eta$ transition carbide. From HEXRD, $\eta$ transition carbide and some cementite were identified. Again, it is possible that the small amounts of cementite may be due to incomplete austenitization (e.g., Figure 5), or perhaps forms during the quench (autotempering) from the austenitizing temperature. That said, early stage (paraequilibrium) cementite at these low temperatures would not be expected to show element partitioning, as noted previously, given that $\mathrm{Si}$ partitioning with respect to the carbide and matrix was not observed until $325^{\circ} \mathrm{C}$ in previous work. ${ }^{[14]}$ 


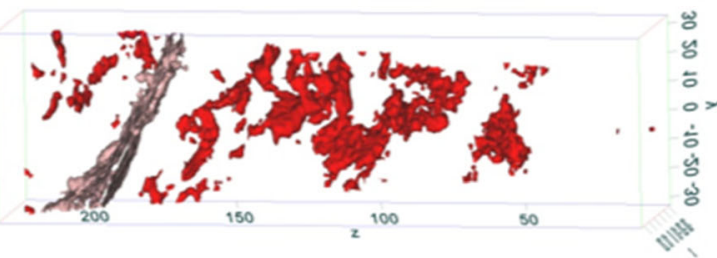

(a)

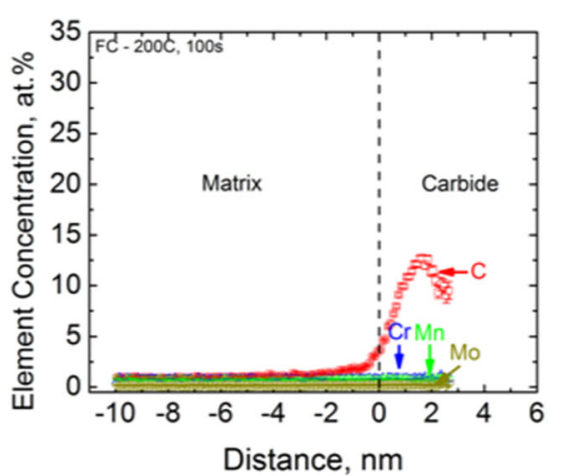

(b)

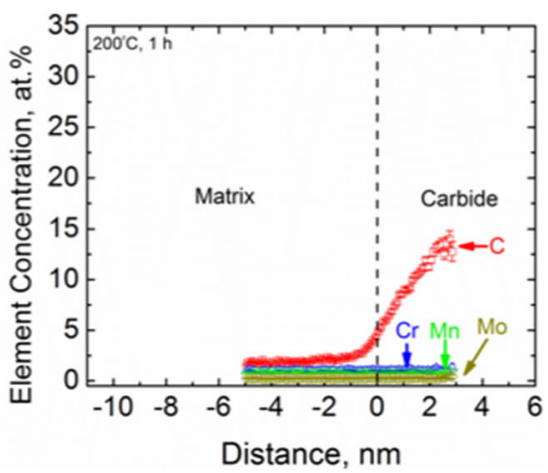

(d)

Fig. 8-(a) An APT C map (scale in nms) with 5 at. pct C iso-concentration surfaces of 4340 steel after fast quenching in the dilatometer and tempering at $200{ }^{\circ} \mathrm{C}$ for $100 \mathrm{~s},(b)$ a proximity histogram of the region highlighted in (a), (c) an APT C map (scale in nms) with 5 at. pct C iso-concentration surfaces of 4340 steel after oil quenching and tempering at $200{ }^{\circ} \mathrm{C}$ for $1 \mathrm{~h}$, and $(d)$ a proximity histogram of the region highlighted in (c). (c) Reprinted from Ref. [54] (Color figure online).

Consideration of potential carbide formation in austenite may also be warranted, as also noted previously. ${ }^{[29]}$

\section{E. Microstructure After $Q \& T$ at $300^{\circ} \mathrm{C}$}

TEM and APT comparing fast and slow dilatometric quenching, followed by short-time tempering at $300{ }^{\circ} \mathrm{C}$ for 100 seconds are provided in Figure 10, along with similar results after oil quenching and tempering at 300 ${ }^{\circ} \mathrm{C}$ for 1 hour. Recall from Figure 2 that fast/slow quenching and tempering at $300{ }^{\circ} \mathrm{C}$ for 100 seconds are the only conditions in this work that exhibit significant volume changes measured by dilatometry. Figure 10(a) reveals the carbides after fast quenching and tempering at $300{ }^{\circ} \mathrm{C}$ for 100 second in an off-zone axis BF TEM image, accompanied by [113] $]_{\alpha}$ (Figure $10(\mathrm{~b})$ ) and $[011]_{\alpha}$ (Figure 10(c)) zone axis SADPs and schematics. The phases can be indexed as ferrite/martensite and $\eta$ transition carbide. Magnetite surface oxide is also observed in the electron diffraction patterns. Complementary APT in Figures 10(d) and (e) shows C-containing, plate-like features without substitutional element partitioning.

Figure 10(f) shows an off-zone axis BF TEM image and Figure $10(\mathrm{~g})$ shows a $[\overline{1} 00]_{\alpha}$ zone axis BF TEM image after slow dilatometric quenching, followed by tempering at $300{ }^{\circ} \mathrm{C}$ for 100 second. Both images and the electron diffraction in Figure $10(\mathrm{~g})$ reveal $\eta$ transition carbides in the tempered martensite matrix. Similar to Figures 10(d) and (e) after fast dilatometric quenching and tempering at $300{ }^{\circ} \mathrm{C}$ for $100 \mathrm{~s}$, slow quenching with the same tempering conditions results in plate-like features observed by APT (Figures 10(h) and (i)), without substitutional element partitioning between the carbide and matrix phases. After oil quenching and conventional tempering at $300{ }^{\circ} \mathrm{C}$ for 1 hour, $\eta$ transition carbides are still found in the microstructure, as shown by the $[011]_{\alpha}$ zone axis BF TEM image in Figure 10(j) and the accompanying [011] $]_{\alpha}$ and [113] $]_{\alpha}$ SADPs in Figures $10(\mathrm{k})$ and (l), respectively. APT of this condition also suggests (paraequilibrium) $\eta$ transition carbide, consistent with TEM and results from short-time tempering at $300{ }^{\circ} \mathrm{C}$.

\section{F. Microstructure After $Q \& T$ Above $300{ }^{\circ} \mathrm{C}$}

Although TEM and APT after short-time (100 second) and conventional tempering at $300{ }^{\circ} \mathrm{C}$ reveals primarily $\eta$ transition carbide, HEXRD also provides supporting evidence for the existence of Hägg or $\chi$ carbide, as shown in Figures 11(a) and (b). HEXRD after oil quenching and tempering at $325^{\circ} \mathrm{C}$ for 2 hour is also shown in Figure 11(c), indicating the presence of Hägg transition carbide, albeit at a reduced amount. The Hägg transition carbide appears to form between $300{ }^{\circ} \mathrm{C}$ and $325^{\circ} \mathrm{C}$, and is present after tempering for 


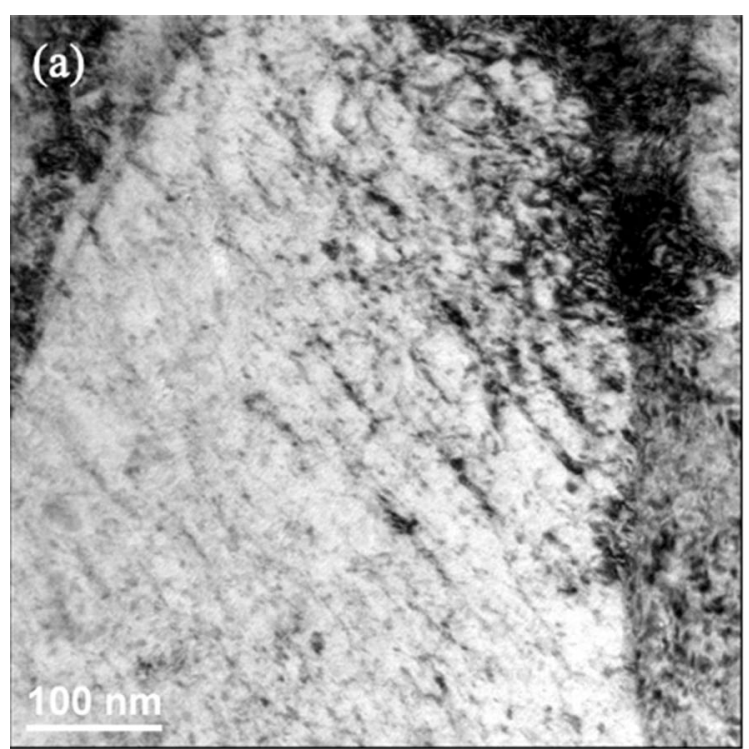

(d)

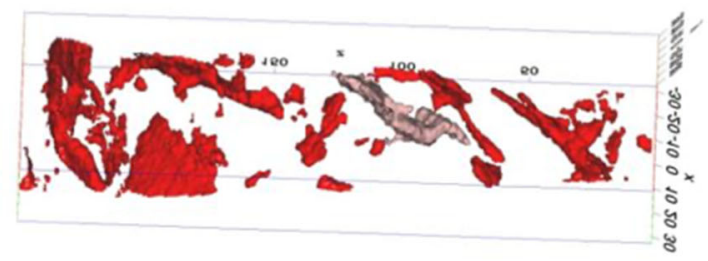

(f)

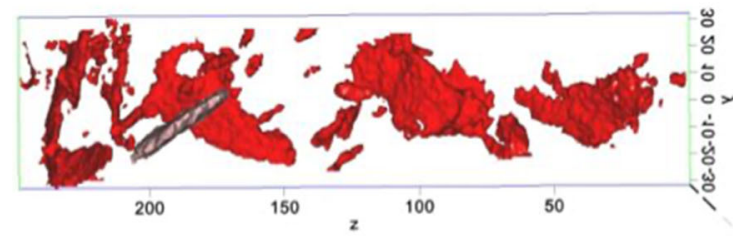

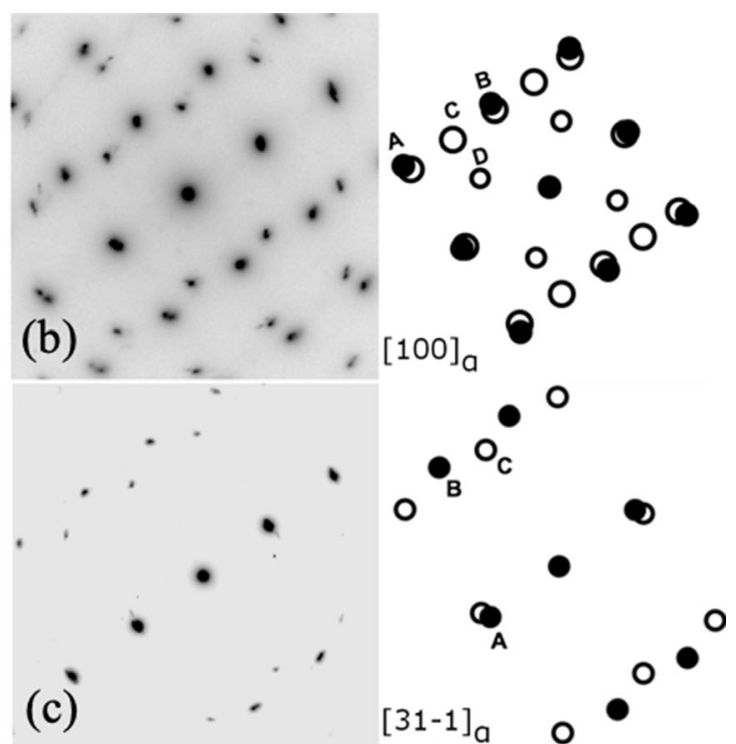
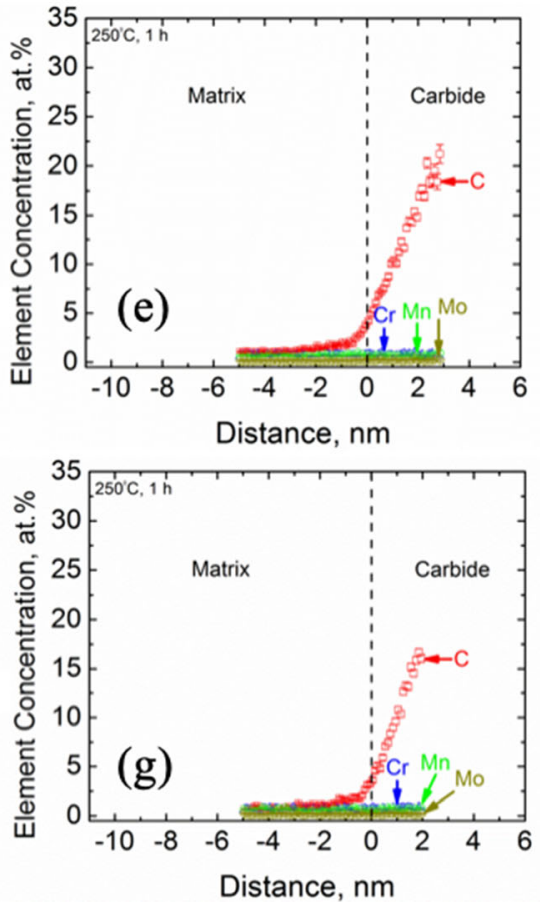

Fig. 9- (a) BF TEM image (off zone) of $\eta$ transition carbides in 4340 steel after oil quenching and tempering at $250{ }^{\circ} \mathrm{C}$ for $1 \mathrm{~h}$, (b) SADP of a $[100]_{\alpha}$ zone axis; $\alpha=(020)_{\alpha}, B=(01 \overline{1})_{\alpha}, C=(210)_{\eta}$, and $D=(110)_{\eta}$ (surface oxide, magnetite, can also contribute to the intensity shown at this g-vector). (c) SADP of a $[31 \overline{1}]$ zone axis; $\alpha=(0 \overline{2})$ and $(0 \overline{2} 0), B=(1 \overline{2} 1)$, and $C=(\overline{3} 01),(d)$ an APT C map (scale in nms) with 5 at. pct $\mathrm{C}$ iso-concentration surfaces of 4340 steel after oil quenching and tempering at $250{ }^{\circ} \mathrm{C}$ for $1 \mathrm{~h},(e)$ a proximity histogram of the region highlighted in (e), $(f)$ an APT C map (scale in nms) with 5 at. pct $\mathrm{C}$ iso-concentration surfaces of 4340 steel after oil quenching and tempering at $250{ }^{\circ} \mathrm{C}$ for $1 \mathrm{~h}$, and $(\mathrm{g})$ a proximity histogram of the region highlighted in (f) (Color figure online).

times up to 2 hours, although further studies are needed to understand the window of formation and precipitation kinetics, especially at short times. TEM and MES studies on the tempering of Fe-1C (wt pct) alloys have indicated the presence of Hägg or $\chi$ carbide after tempering between $250{ }^{\circ} \mathrm{C}$ and $400{ }^{\circ} \mathrm{C}$, where this transition carbide is observed in the beginning stages of cementite formation. ${ }^{[26,27]}$ Based upon the results presented here, the transition from $\eta$ carbide to cementite appears to happen between $300{ }^{\circ} \mathrm{C}$ and $325^{\circ} \mathrm{C}$, for both conventional and short-time tempering treatments. In addition to the trends observed with respect to transition carbides, an appreciable change in the relative austenite peak intensities is also observed in Figure 11. Slow quenching qualitatively results in a higher austenite fraction than fast quenching for a given tempering treatment (Figures 11(a) and (b)); this trend is also observed in the HEXRD in Figure 4 after fast and slow quenching. Oil quenching and tempering at $325^{\circ} \mathrm{C}$ for 2 hour results in the lowest austenite amount, as expected, 

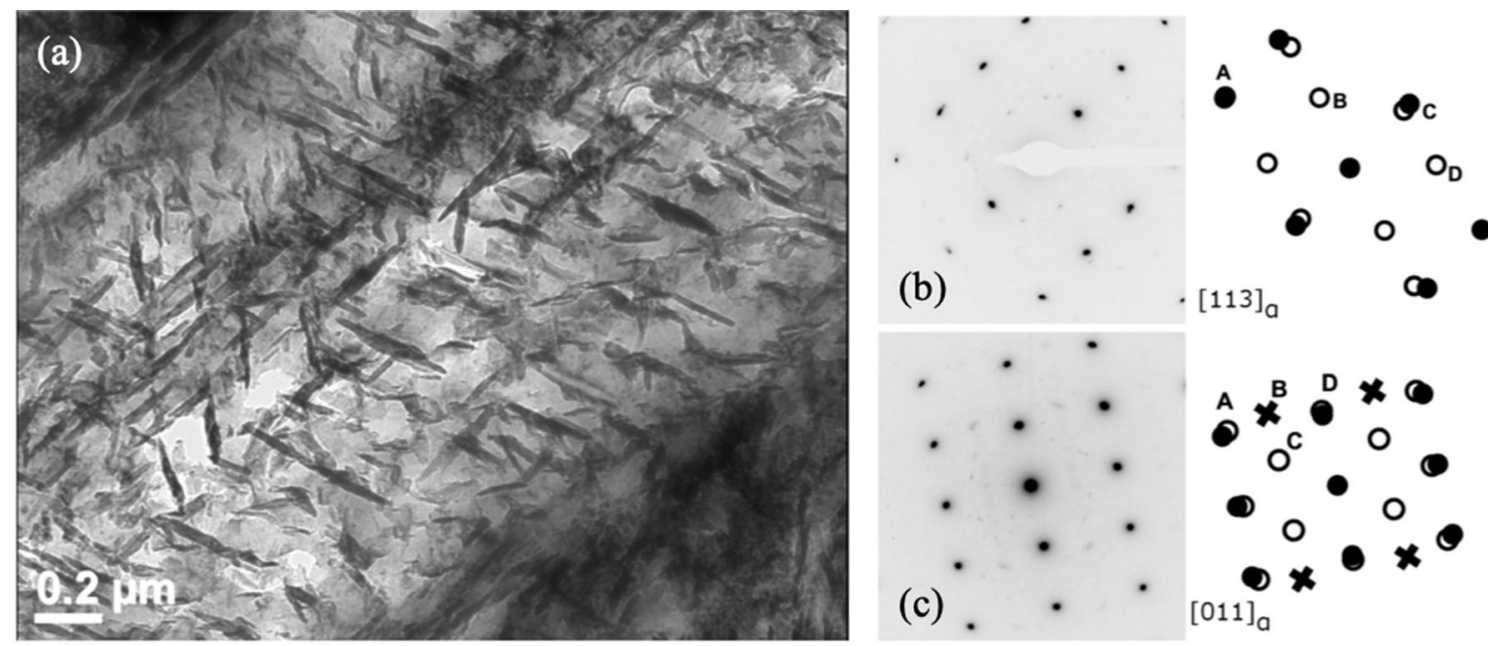

(d)
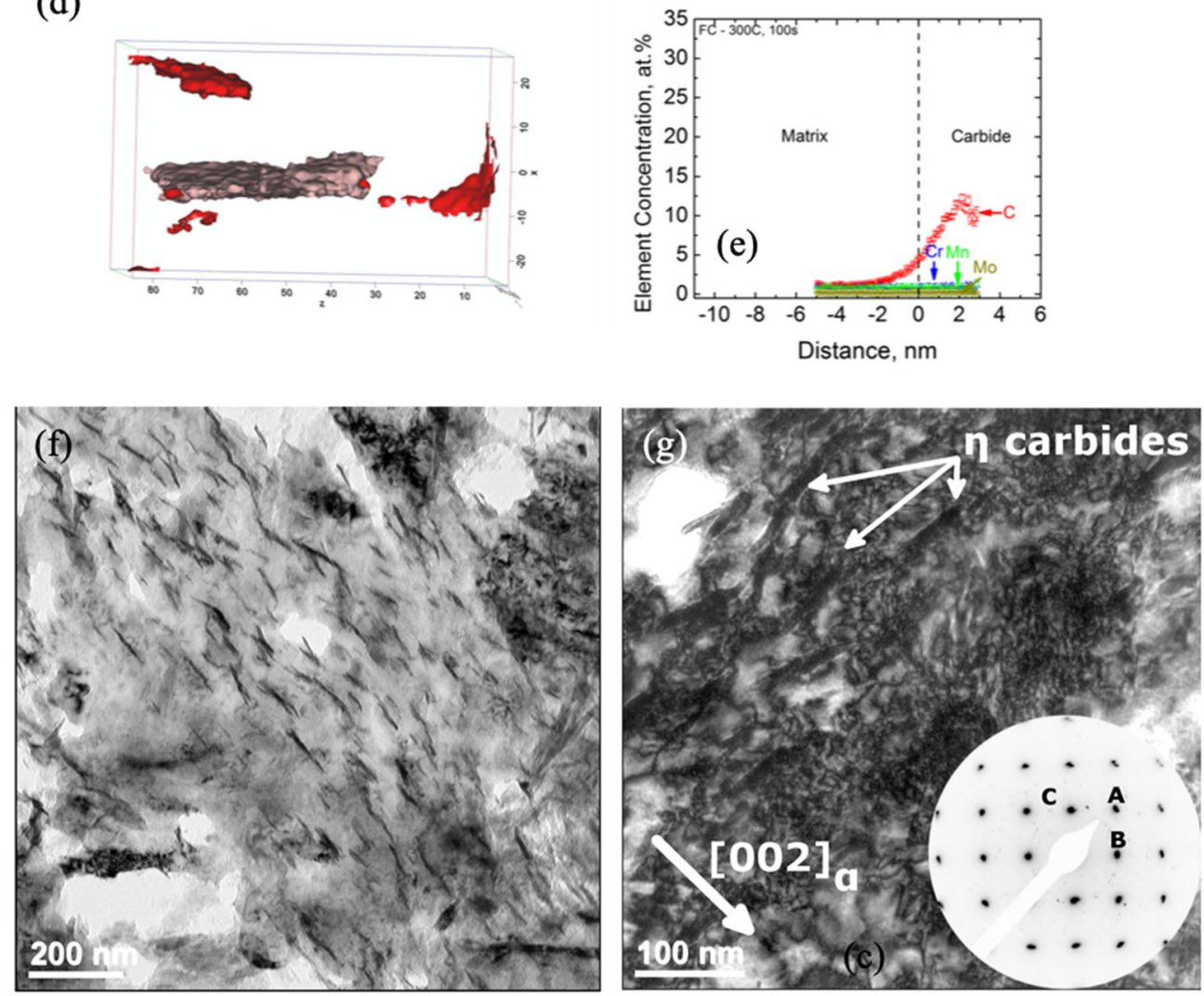

(h)

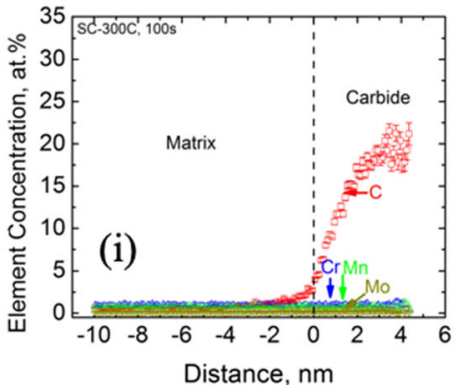


4 Fig. 10-(a) BF TEM image (off-zone) of $\eta$ transition carbides after fast quenching 4340 steel in the dilatometer and tempering at $300{ }^{\circ} \mathrm{C}$ for $100 \mathrm{~s},(b)$ SADP of a $[113]_{\alpha}$ zone axis; $\alpha=(\overline{1} 21)_{\alpha}, B=(\overline{1} 0 \overline{1})_{\eta_{2}}$, $C=(\overline{1} 10)_{a}$, and $D=(210)_{\eta_{2}},(c)$ SADP of a $[011]_{\alpha}$ zone axis; $\alpha \stackrel{\eta_{2}}{=}$ $(\overline{2} 1 \overline{1}), B \stackrel{a}{=} X=\{440\}_{O}$ (surface oxide, magnetite) (intensities for other oxide reflection may be present but not marked in schematic), $C=(10 \overline{1})_{\eta}$, and $D=(00 \overline{1})_{a}$ and $(00 \overline{2})_{\eta_{2}},(d)$ an APT C map (scale in nms) with 5 at. pct $C$ iso-concentration surfaces of 4340 steel after fast quenching in the dilatometer and tempering at $300{ }^{\circ} \mathrm{C}$ for $100 \mathrm{~s}$, (e) a proximity histogram of the region highlighted in (d), (f) $\mathrm{BF}$ TEM image (off zone) of $\eta$ transition carbides in 4340 steel in a martensitic lath after slow quenching in a dilatometer and tempering at $300{ }^{\circ} \mathrm{C}$ for $100 \mathrm{~s}$ and $(g)$ a BF TEM image (off-zone) with a properly rotated SAD pattern of the $[\overline{100}]_{\alpha}$ zone axis; $A=(020)_{\alpha}$, $B=(011)_{\alpha}$, and $C=(2 \overline{1} 0)_{\eta},(h)$ an APT C map(scale in nms) with 5 at. pet $C$ iso-concentration surfaces of 4340 steel after slow quenching in the dilatometer and tempering at $300{ }^{\circ} \mathrm{C}$ for $100 \mathrm{~s}$, (i) a proximity histogram of the region highlighted in $(\mathrm{h}),(j)[011]_{\alpha}$ zone axis BF TEM of $\eta$ transition carbides in 4340 steel after oil quenching and tempering at $300{ }^{\circ} \mathrm{C}$ for $1 \mathrm{~h},(k)$ properly rotated SADP of a $[011]_{\alpha}$ zone axis; $\alpha=(01 \overline{1})_{\alpha}, B=(\overline{200})_{a}, C=(30 \overline{1})_{\eta_{2}}$ and $X=\{440\}_{O}$ (surface oxide, magnetite) (intensities for other oxide reflection may be present but not marked in schematic), and $D$ $=(10 \overline{1})_{\eta},(l)$ SADP of a $[113]_{\alpha}$ zone axis (not rotated); $\alpha=(\overline{2} \overline{1} 1)_{\alpha}$, $B=\left(11^{1} 0\right)_{a}, C=(210)_{\eta_{2}}$, and $D=(101)_{\eta_{2}},(m)$ an APT C map (scale in nms) with 5 at. pct $C$ iso-concentration surfaces of 4340 steel after oil quenching and tempering at $300{ }^{\circ} \mathrm{C}$ for $1 \mathrm{~h}$, and $(n)$ a proximity histogram of the region highlighted in $(\mathrm{m})$. (f, g) Reprinted from Ref. [54] (Color figure online). given the extent of anticipated austenite decomposition for this time/temperature combination.

In a previous study of high temperature tempering of the same 4340 steel studied here at temperatures above $325{ }^{\circ} \mathrm{C}$, faulted cementite (i.e., cementite with internal structure) was observed by TEM (Figure 12). ${ }^{[14]}$ Selected area diffraction and microdiffraction were used to determine the Bagaryatsky orientation relationship between the cementite and matrix. At $575{ }^{\circ} \mathrm{C}$ for 2 hours, TEM showed indications of cementite spheroidization and loss of this orientation relationship. ${ }^{[14]}$

Figure 13 shows a classic figure, originally produced by Williamson et al., that highlights the microstructural changes associated with the different stages of tempering in 4340 steel. $^{[7]}$ Their results have been replotted together with selected austenite, cementite, and $\eta$ transition carbide amounts determined from MES in this work. Total $\eta$ transition carbide is shown, in addition to stoichiometric and non-stoichiometric amounts of $\eta$ transition carbide. The identification of stoichiometric and non-stoichiometric $\eta\left(\eta_{\mathrm{s}}\right.$ and $\left.\eta_{\mathrm{ns}}\right)$ was described in Reference 52 based on the observation of two subspectra with distinct magnetic hyperfine fields, at about 17 and $25 \mathrm{~T}$, respectively. These values were found here
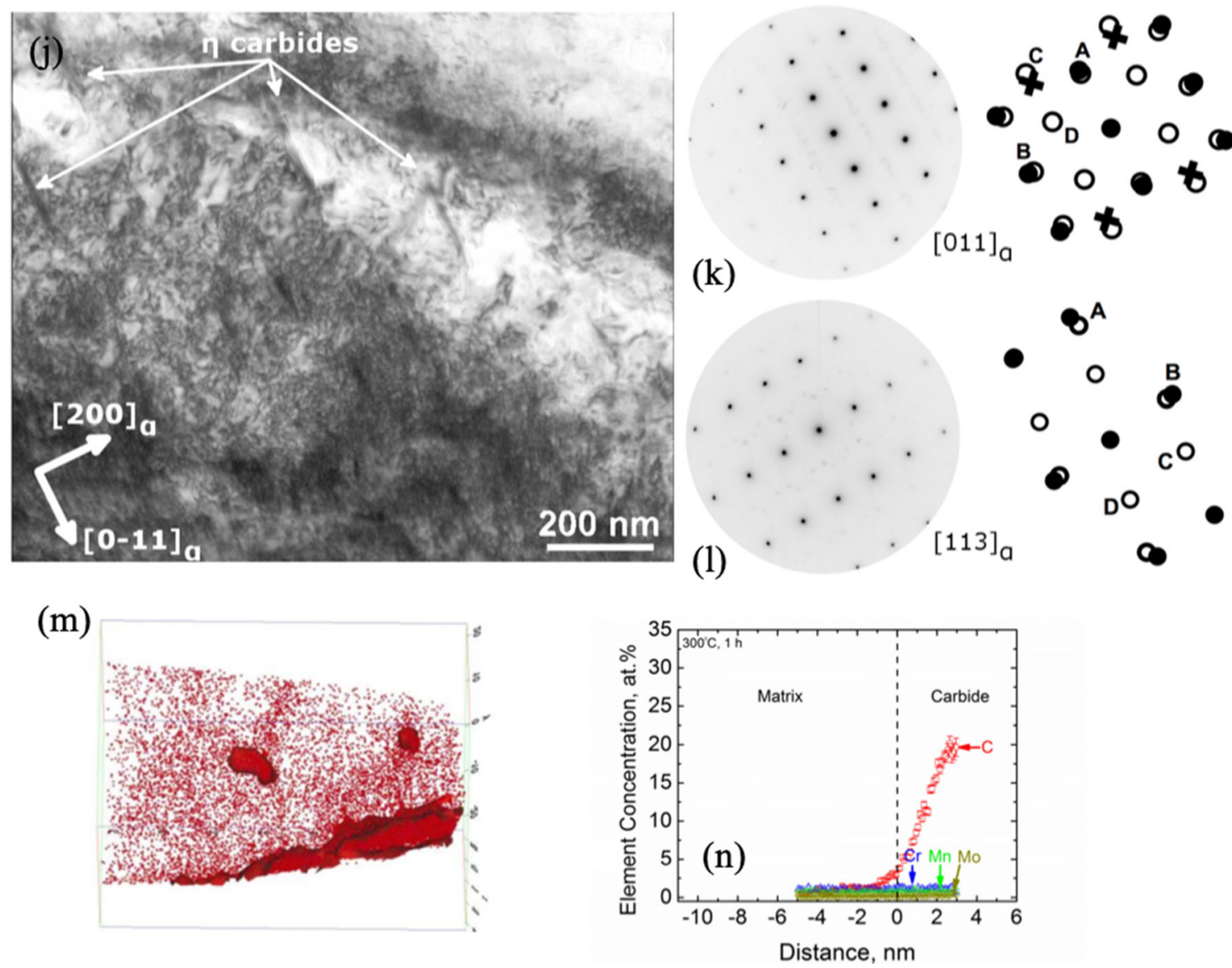

Fig. 10 - continued. 


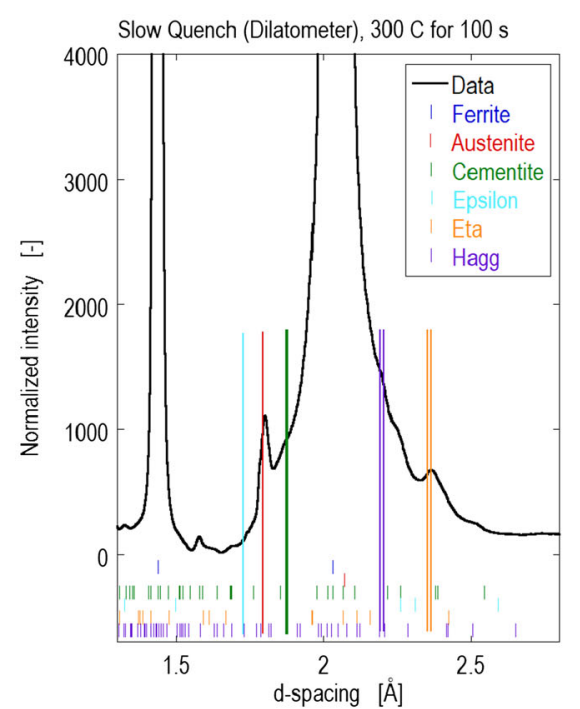

(a)

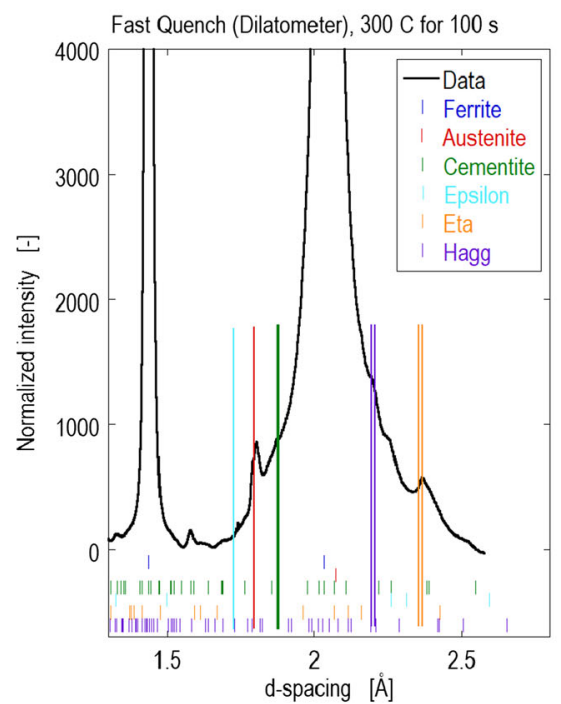

(b)

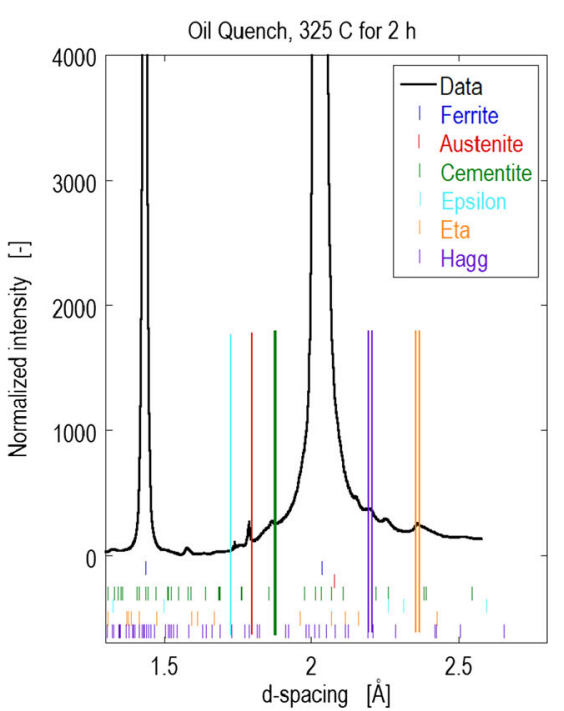

(c)

Fig. 11-HEXRD of 4340 steel after (a) fast quenching in the dilatometer and tempering at $300{ }^{\circ} \mathrm{C}$ for $100 \mathrm{~s}$, (b) slow quenching in the dilatometer and tempering at $300{ }^{\circ} \mathrm{C}$ for $100 \mathrm{~s}$, and $(c)$ oil quenching and tempering at $325{ }^{\circ} \mathrm{C}$ for $2 \mathrm{~h}$. Note the presence of Hägg transition carbide in all conditions. Tick marks highlight peak positions for the relevant phases, and the larger stick markings indicate peak positions of specific phases surrounding the high intensity ferrite peak to facilitate phase identification (Color figure online).

also for the 4340 steel. These can be ascribed to regions of $\eta-\mathrm{Fe}_{2} \mathrm{C}$ with all $\mathrm{Fe}$ atoms having $3 \mathrm{C}$ nearest neighbors and $\mathrm{C}$ - deficient regions of $\eta-\mathrm{Fe}_{3} \mathrm{C}$ where the $\mathrm{Fe}$ has $2 \mathrm{C}$ nearest neighbors. The $\mathrm{C}$ nearest neighbors play the strongest role in controlling the magnetic field. Although the cementite has a hyperfine field $(\sim 18$ to 19 $\mathrm{T}$ ), similar to that of the $\eta_{\mathrm{s}}$ transition carbide, differences in the three spectral parameters of isomer shift, quadrupole splitting, and magnetic hyperfine field ${ }^{[52,53]}$ enable distinction of cementite from $\eta_{\mathrm{s}}$. However, increased uncertainty of the relative amounts of $\eta_{\mathrm{s}}$ and cementite occurs if they are both present in similar quantity, such as shown in Figure 3(d) of Reference 53. Figure 13 shows that the tempering regions for $\eta$-carbide and cementite are separated below and above about $300{ }^{\circ} \mathrm{C}$, respectively. The detection of Hägg carbide is difficult by MES due to its complex, multi-component spectrum $^{[26]}$ and the narrow range of its appearance, as indicated in Figure 13. It can be seen, in general, that the main carbide present upon tempering below $300{ }^{\circ} \mathrm{C}$ is $\eta$ transition carbide, and the majority is stoichiometric. As noted previously, these results suggest that obtaining representative measurements of the fine-scale $\mathrm{C}$-containing features produced after quenching and LTT by APT is challenging.

Of particular note in Figure 13 is the clear decrease in austenite amount, along with the concomitant increase in cementite amount in the $200{ }^{\circ} \mathrm{C}$ to $300{ }^{\circ} \mathrm{C}$ temperature range associated with the second stage of tempering. These behaviors signify the decomposition of retained austenite to ferrite and cementite, although note that a substantial fraction of $\eta$ transition carbide is still present in the microstructure. From $250{ }^{\circ} \mathrm{C}$ to 350 ${ }^{\circ} \mathrm{C}$ during the third stage of tempering, the replacement of transition carbides, identified in this work as $\eta$ transition carbide, rather than $\varepsilon$ transition carbide, and low-carbon martensite by cementite and ferrite occurs. In addition to the results of Williamson et al. and the MES results obtained here, the temperature window determined by HEXRD where Hägg transition carbide occurs is also indicated in Figure 13, which falls within the temperature window reported previously. ${ }^{[27,28]}$ Future work to better understand this transition regime from $\eta$ transition carbide to cementite, including the role of alloying effects and kinetics, is warranted.

Although not shown here, APT revealed that early stage cementite forms under paraequilibrium conditions, without substitutional element partitioning, ${ }^{[14]}$ in agreement with previous studies. ${ }^{[15,36,39,40]} \mathrm{Si}$ redistribution was found to occur after tempering at $325{ }^{\circ} \mathrm{C}$ for 2 hours, where $\mathrm{Si}$ starts to be rejected from the cementite into the matrix. Tempering at $425^{\circ} \mathrm{C}$ and $575{ }^{\circ} \mathrm{C}$ results in not only $\mathrm{Si}$ being rejected from the cementite, but similar trends were also observed for $\mathrm{Al}$ and $\mathrm{Ni}^{\left[{ }^{[14]}\right.} \mathrm{Cr}$, $\mathrm{Mo}$, and Mn were also reported to partition within the cementite, particularly near cementite/matrix interfaces. Similar APT results to those published in Reference 14 are shown in Figure 14 after tempering at $575^{\circ} \mathrm{C}$ for 2 hours. Partitioning of $\mathrm{Cr}, \mathrm{Mn}$ and Mo to the cementite near the cementite/matrix interface is observed, and $\mathrm{Si}$ is rejected from the cementite. The $\mathrm{C}$ level of larger cementite plates such as that shown in Figure 14 is approximately 25 at. pct, or the anticipated level for cementite. A comment may be warranted regarding the partitioning of $\mathrm{Cr}, \mathrm{Mo}$, and $\mathrm{Mn}$ observed in Figure 14, as compared to that shown in Figure 6 for $\mathrm{M}_{23} \mathrm{C}_{6}$. Note the substantially higher levels of $\mathrm{Cr}$ (greater than 15 and nearing 20 at. pct) associated with $\mathrm{M}_{23} \mathrm{C}_{6}$, the relatively high Mo levels ( $~ 5$ at. pct), and the Mn levels of $\sim 2.5$ at. pct in Figure 6 with "flat" concentration profiles into the carbide, whereas the cementite in Figure 14 has $\sim 10$ at. pct $\mathrm{Cr}$, higher Mn levels ( 5 at. pct), and reduced 

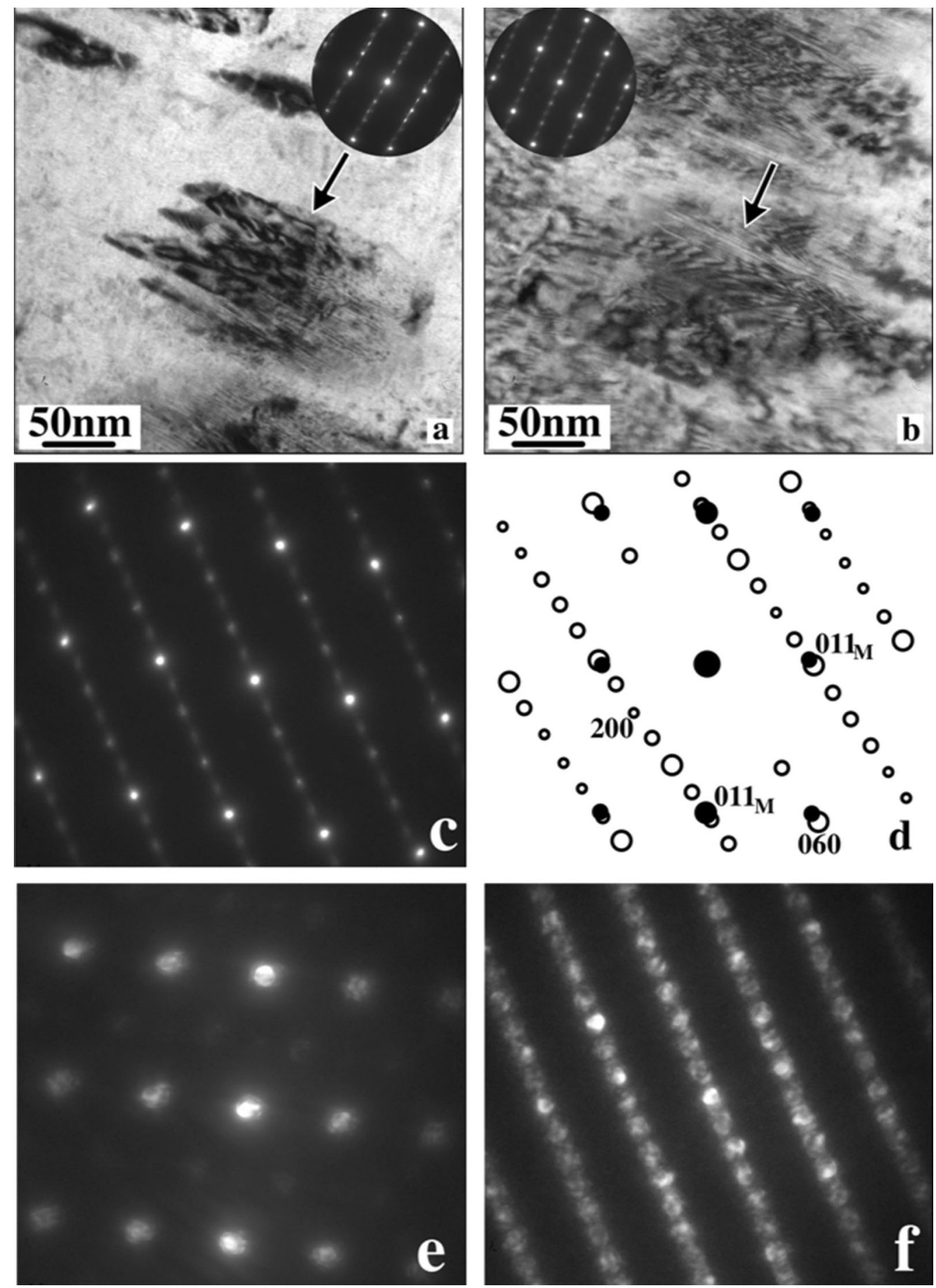

Fig. 12 - Cementite with internal structure (arrows) and selected area diffraction for $(a)$ the $[\overline{11} 3]_{\alpha}$ zone axis and $(b)$ the $[0 \overline{1} 1]_{\alpha}$ zone axis after oil quenching and tempering 4340 steel at $325^{\circ} \mathrm{C}$ for $2 \mathrm{~h}$. (c) Selected area diffraction for the $[0 \overline{1} 1]_{\alpha}$ zone axis and (d) a simulated electron diffraction pattern for the matrix (filled circles) and cementite (open circles) and microdiffraction for $(e)$ the matrix and $(f)$ cementite used to determine the Bagaryatsky orientation relationship. Reprinted from Ref. [14] with permission from Elsevier.

Mo levels within the cementite, primarily near the cementite/matrix interface.

After high temperature tempering, evolved cementite is clearly present in the microstructure (e.g., Figure 14), which is also shown in Figures 15(a) through (c) of cementite from the same low- and high-P steels studied by Materkowski and Krauss ${ }^{[8]}$ that was re-heat treated and analyzed in this work. Recall the Charpy impact properties of these steels were presented in Figure 1(b). APT of this low-P steel heat-treated in the current work shows the distribution of C, Figure 15(a), and P, Figure $15(\mathrm{~b})$. A proximity histogram of the 5 at. pct $\mathrm{C}$ iso-concentration surface highlighted in Figure 15(a) shows redistribution of $\mathrm{Cr}$, Mo, and $\mathrm{Mn}$ to the 


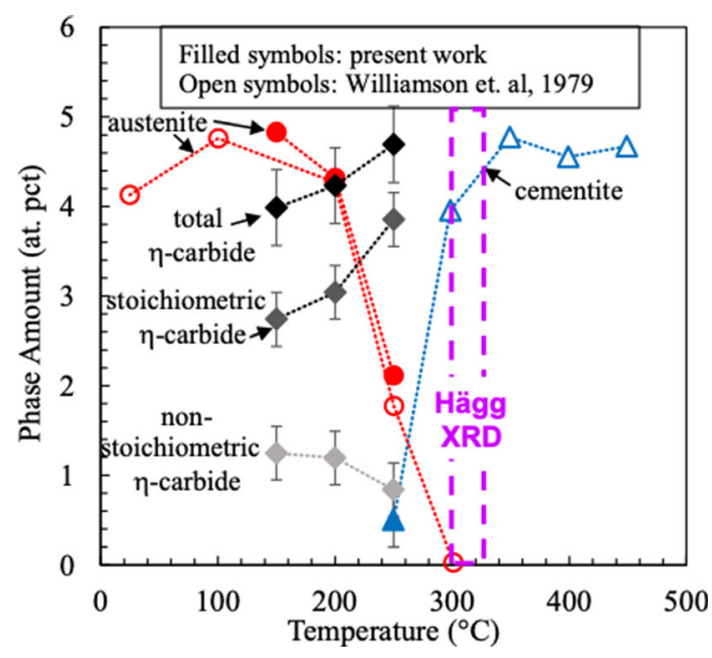

Fig. 13-Phase amount vs. tempering temperature (1 $\mathrm{h}$ tempering treatment) in 4340 steel as determined from MES and the region where Hägg carbide was identified by HEXRD (current work), along with previous results from Williamson et al. ${ }^{[7]}$ (Color figure online).

cementite near the cementite/matrix interface, whereas $\mathrm{Si}$ is rejected from the cementite, consistent with the results shown in Figure 14 and in previous work by Clarke et al. ${ }^{[14]}$. Figures $15(\mathrm{~d})$ and (e) show the $\mathrm{C}$ and $\mathrm{P}$ maps in the high-P steel of Materkowski and Krauss, ${ }^{[8]}$ while Figure 15(f) shows P segregation to cementite/matrix interfaces in the 4340 steel studied here that contains 0.01 wt pct. $P{ }^{[14]}$ or a medium level of $\mathrm{P}$ compared to the steels studied by Materkowski and Krauss. ${ }^{\text {[8] }}$ These results suggest that $\mathrm{P}$ segregation to cementite/matrix interfaces and/or prior austenite grain boundaries ${ }^{[8,10-13]}$ may be significant and play a role in reducing fracture resistance in these steels. Previous work after tempering at $575{ }^{\circ} \mathrm{C}$ for 2 hours also suggests that $\mathrm{P}$ segregation to cementite/matrix interfaces in 4340 may play a role as a contributing mechanism for slowing cementite growth and retarding the spheroidization of elongated carbide structures. ${ }^{[14,60,61]}$ This mechanism may be important when considering alloying effects, such as increased Si content found in 300-M, used to defer the formation of cementite to longer times and higher tempering temperatures, particularly when employing rapid tempering treatments, as discussed later.

\section{G. Effects of Quench Rate on Autopartitioning and Implications for 3rd Generation AHSS}

In addition to the evolution of carbon-clustering, transition carbides, and cementite, the amount of retained austenite also varies with quench rate and subsequent tempering. ${ }^{[10,28,62]}$ Figure 16(a) from Sherman et al. shows results from MES, indicating retained austenite amount in the as-quenched condition decreases with increasing quench rate in a 4130-type steel with elevated Si content $(1.5 \mathrm{wt}$ pct $) .{ }^{[28]}$ Similarly, the austenite amount determined here from HEXRD in 4340 steel is lower for the fast quench rate condition, Figure 16(b), relative to that produced by slow quenching. The lower austenite amounts persist through short-time tempering at $100{ }^{\circ} \mathrm{C}, 200{ }^{\circ} \mathrm{C}$, and $300{ }^{\circ} \mathrm{C}$, suggesting that carbon partitioning, or "autopartitioning", to austenite occurs during quenching; and, greater partitioning occurs during slow quenching. Early work by Sarikaya et al. showed increased carbon partitioning at austenite/martensite interfaces after oil quenching and $200{ }^{\circ} \mathrm{C}$ tempering compared to that produced by water quenching. ${ }^{[62]}$ Thus, autopartitioning may be a useful approach to retain greater amounts of stabilized retained austenite that impact subsequent martensite tempering reactions like austenite decomposition, which may be important to mechanical properties. ${ }^{[10,44,45]}$

The presence of significant fractions of retained austenite is a central microstructure design strategy for another class of steels, the $3^{\text {rd }}$ generation AHSS, employing TRansformation Induced Plasticity (TRIP) to improve strength-ductility combinations in auto-body sheet steels, ${ }^{[63-66]}$ and was an early objective of the Quenching \& Partitioning (Q\&P) process. ${ }^{[64,66]}$ Q\&P seeks to retain significant fractions of carbon-enriched austenite in the final microstructure by performing novel thermal cycling to partition carbon to retained austenite during a partitioning step at a partitioning temperature (PT), just after quenching to a temperature (QT) between $M_{\mathrm{s}}$ and $M_{\mathrm{f}}$, as illustrated schematically in Figure 17. The higher fractions of austenite produced by slow quenching compared to fast quenching shown here to persist throughout tempering for the 4340 steel studied in this work (Figure 16) suggest that increased autopartitioning of carbon to austenite occurred during slow quenching; thus, higher stability austenite is retained. It is conceivable that austenite amounts may be maximized by manipulating the initial quench rate from the austenitizing or intercritical annealing step ${ }^{[64]}$ (whereby ferrite may also form, depending upon the cooling rate employed, which would also serve to potentially promote austenite carbon enrichment), setting the stage for controlling subsequent microstructural evolution, including martensite tempering reactions that compete for carbon during Q\&P. This remains an area fruitful for future research. New knowledge about the subtle microstructural changes that occur during Q\&T by advanced characterization will enable the design of future steel alloying and processing concepts to create modern steel products with martensite/austenite mixtures and desirable properties.

\section{H. Influences of Rapid Tempering on Mechanical Properties}

Mechanical performance such as fracture resistance can also be correlated to variations in Q\&T parameters. Figure 18(a) shows a replotting of the low-P data from Figure 1(b) against the Holloman-Jaffe tempering parameter and superimposes Charpy impact energy results for the 4340 steel used in a related study after tempering for 1 hour (3600 second) in the $200{ }^{\circ} \mathrm{C}$ to $400{ }^{\circ} \mathrm{C}$ TME range, showing good agreement. ${ }^{[44]}$ Figure 18(b) replots the impact energy data for the 1 hour tempered material, and adds data for samples tempered for short times (100, 10, and 1 seconds) with an equivalent Holloman-Jaffe tempering parameter 


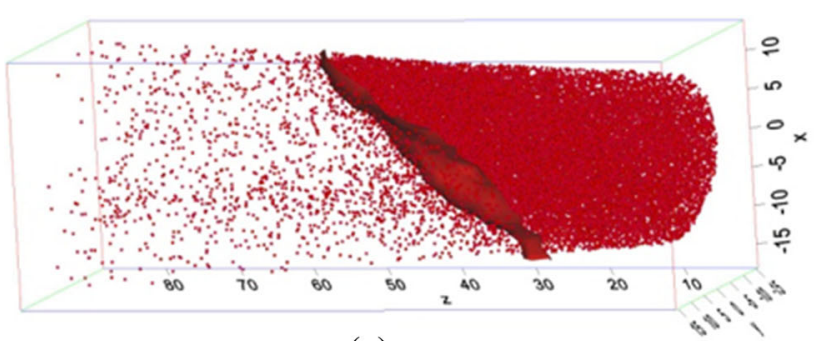

(a)

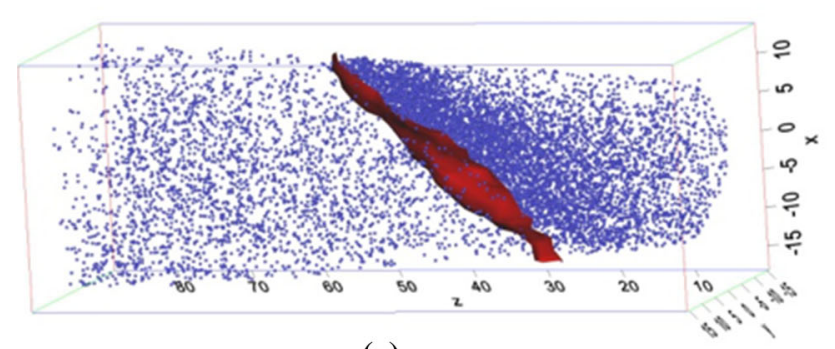

(c)

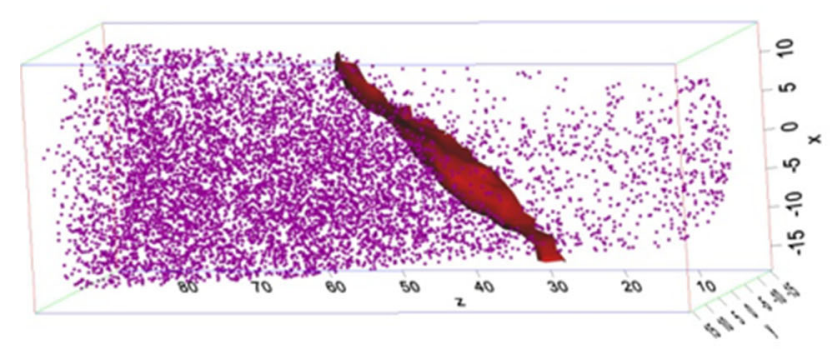

(e)

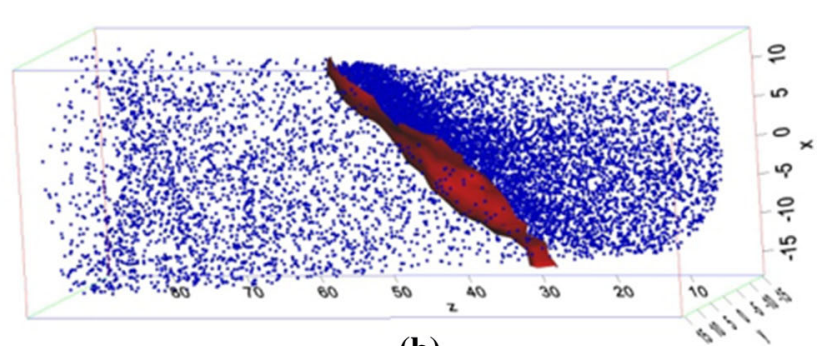

(b)

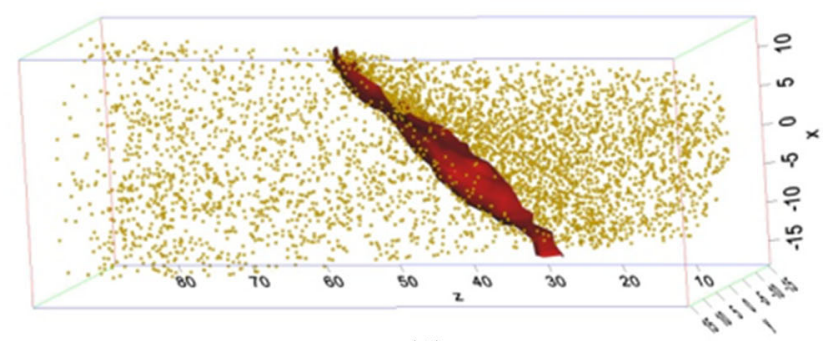

(d)

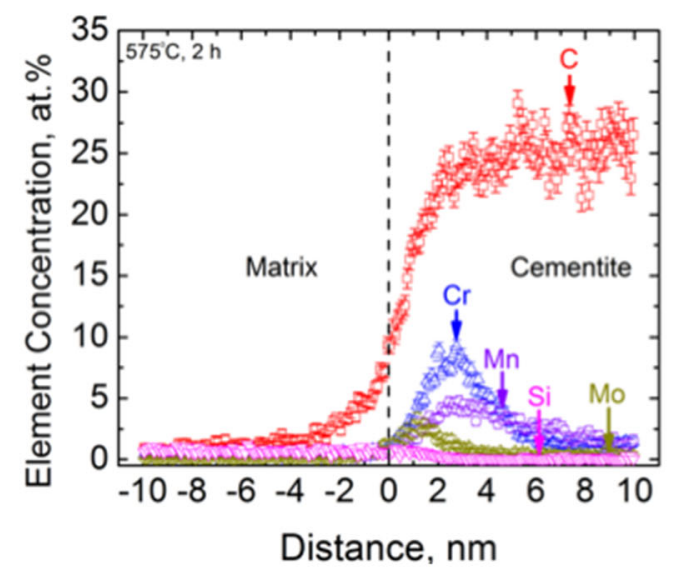

(f)

Fig. 14-APT of the matrix and evolved cementite in 4340 steel after oil quenching and tempering at $575{ }^{\circ} \mathrm{C}$ for $2 \mathrm{~h}$ (scale in $\mathrm{nms}$ ) and $(a)$ an APT C map and a 10 at. pct C iso-concentration surface, $(b)$ an APT $\mathrm{Cr}$ map and the 10 at. pct C iso-concentration surface in (a), $(c)$ an APT $\mathrm{Mn}$ map and the 10 at. pct C iso-concentration surface in (a), (d) an APT Mo map and the 10 at. pct C iso-concentration surface in (a), (e) an APT Si map and the 10 at. pct $\mathrm{C}$ iso-concentration surface in (a), and $(f)$ a proximity histogram for the 10 at. pct $\mathrm{C}$ iso-concentration surface in (a). Reprinted from Ref. [54] (Color figure online).

(i.e., tempered for shorter times with higher maximum temperatures). ${ }^{[44]}$ These rapid tempering heat treatments result in significant increases in the impact energy through the TME range, with the 1 second treatment at a tempering parameter of 13,000 having an impact energy a full $18 \mathrm{~J}$ greater than was measured for a 1 hour conventional tempering treatment. These results show that the low-toughness TME trough can be avoided by performing rapid tempering. Plotting this data relative to a wide range of data from the literature, the results in Figure 18(c) show the significance of this change: at an ultimate tensile strength level of $1.7 \mathrm{GPa}$, the impact energy increases by over 40 pct., and at a toughness level of $30 \mathrm{~J}$, the ultimate tensile strength increases from 1.4 to $2.0 \mathrm{GPa}^{[45]}$ Further detailed characterization work is needed, but initial evaluations of austenite amounts in 4340 steel as a function of rapid tempering treatment are detailed in Figure 18(d), indicating increased retained austenite in all cases for rapid tempering treatments. This is likely due to a difference in the extent of austenite decomposition that occurs during 1 second and 1 hour tempering. The increased fraction of retained austenite from rapid tempering is believed to contribute to the increase in toughness at a given tempering level, promoting the elimination of $\mathrm{TME}^{[44,45]}$ and highlighting future opportunities for rapid thermal processing of martensitic steels. Recent work on rapid tempering of 300-M, which contains higher levels of Si than 4340, resulted in the delay of retained austenite decomposition and cementite precipitation to higher temperatures compared to 4340 steel. $^{[71]}$ Higher peak tempering 


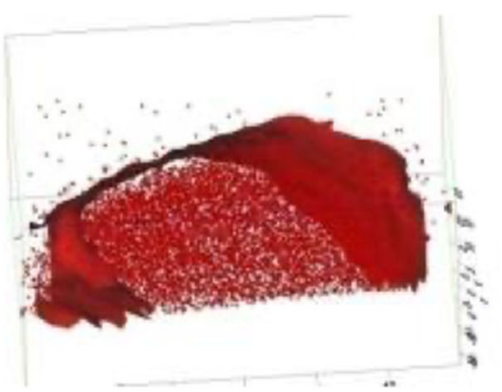

(a)

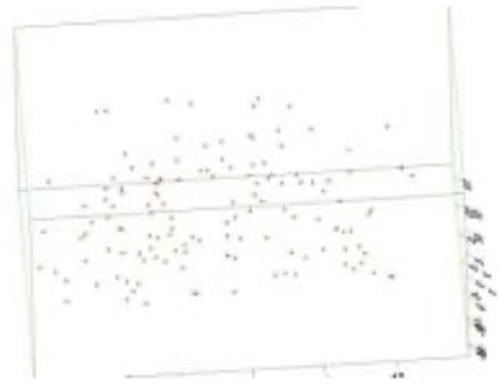

(b)

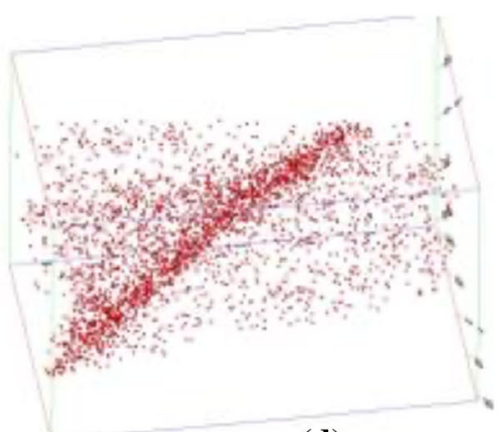

(d)

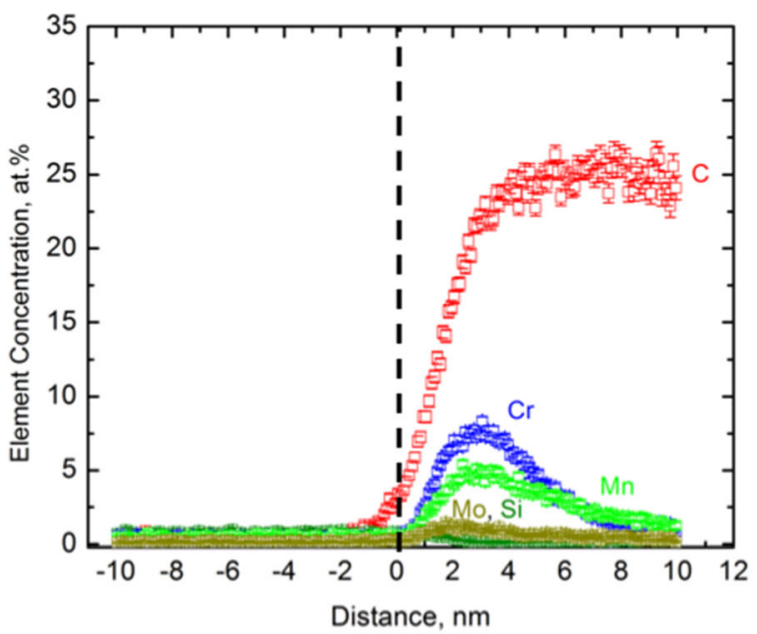

(c)
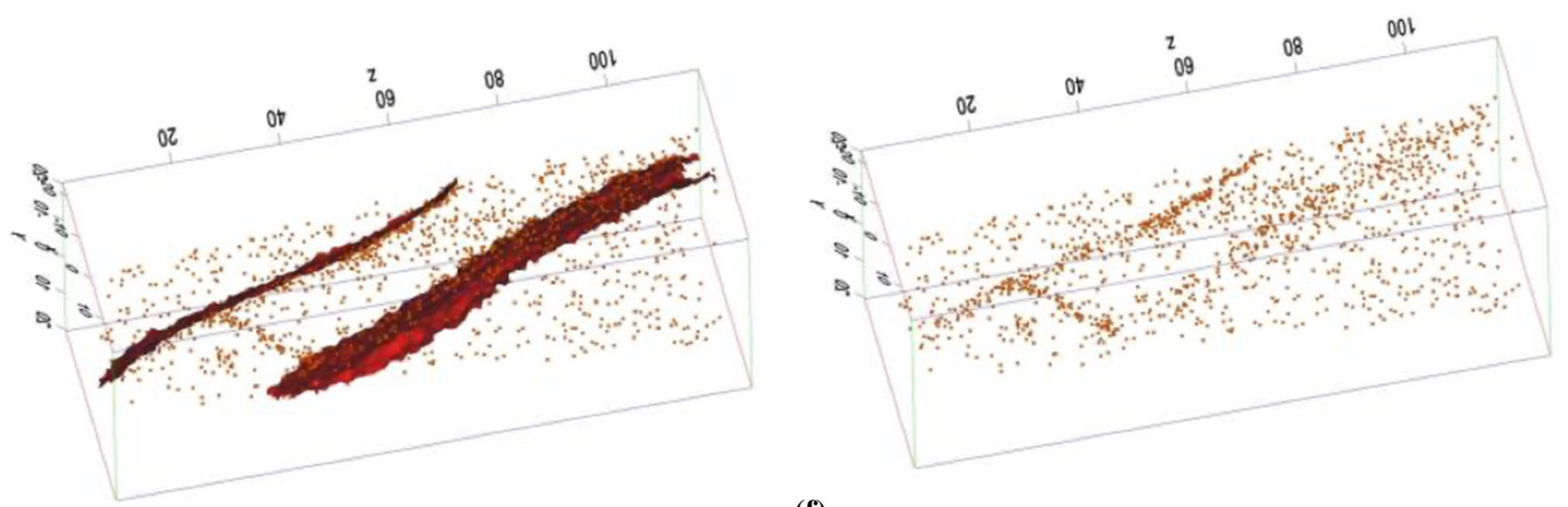

(f)

Fig. 15-(a) An APT C map (scale in nms) and a 5 at. pct iso-concentration surface, $(b)$ an APT P map, and $(c)$ a proximity histogram for the surface highlighted in (a) for a low P $\left(0.003 \mathrm{wt}\right.$ pct) 4340 steel previously studied by Materkowski and Krauss ${ }^{[8]}$ and re-examined here after tempering at $575{ }^{\circ} \mathrm{C}$ for $2 \mathrm{~h},(d)$ an APT C map (scale in nms) and (e) an APT P map (scale in nms) for a high P (0.03 wt pct) 4340 steel previously studied by Materkowski and Krauss ${ }^{[8]}$ and re-examined here after tempering at $575^{\circ} \mathrm{C}$ for $2 \mathrm{~h}$. Note the presence of $\mathrm{P}$ at an interface for the steel with an elevated $\mathrm{P}$ level in (e). $(f) \mathrm{P}$ segregation at cementite/matrix interfaces (defined by 10 at. pct. C iso-concentration surfaces in red) for the $0.01 \mathrm{wt}$ pct P 4340 steel studied here after tempering at $575{ }^{\circ} \mathrm{C}$ for $2 \mathrm{~h}$, reprinted from Ref. [14] with permission from Elsevier (Color figure online).

temperatures are typical during rapid tempering, so other factors that play a role at higher tempering temperatures, such as $\mathrm{P}$ segregation and its interaction with the effects of $\mathrm{Si}$, may warrant further attention. These issues could have important influences on temper embrittlement and mechanical properties in steels like 300-M.
Significant opportunity exists to further understand the role of alloying on microstructural evolution and control during Q\&T. Novel strategies for alloying, coupled with controlled quench rates and/or rapid tempering via processes like induction heating tailored to industrial needs, may afford reduced processing times, increased energy efficiency, improved 


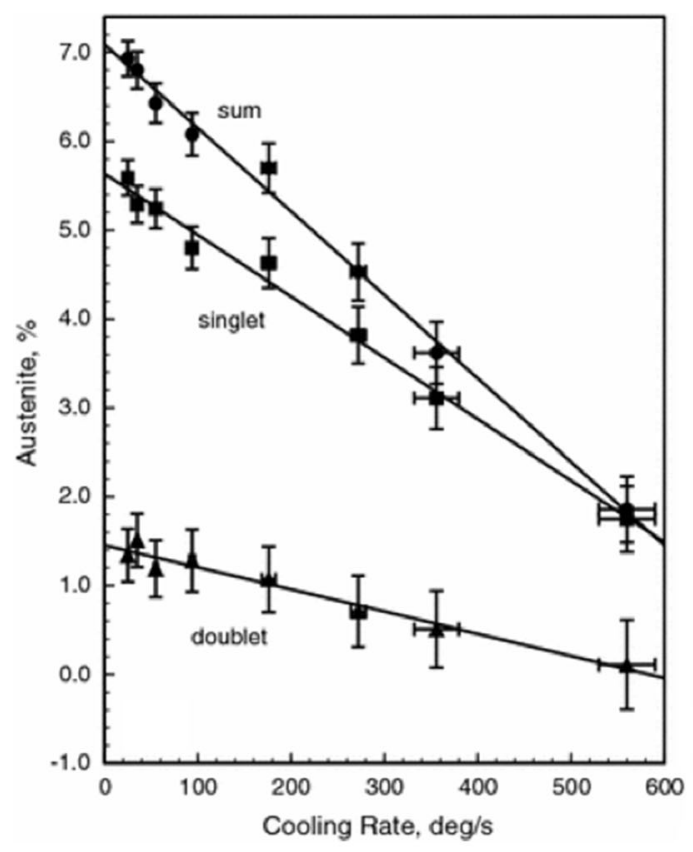

(a)

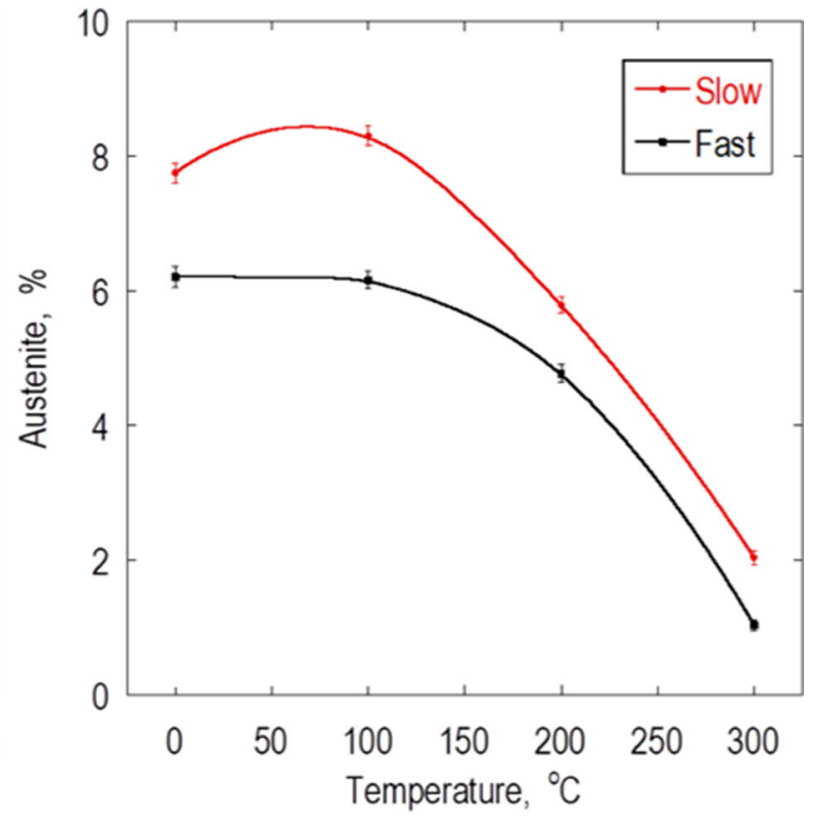

(b)

Fig. 16 - (a) Austenite amount vs. cooling rate in 4340 steel. Reprinted from Ref. [28]. (b) Austenite amounts determined by HEXRD after fast or slow quenching in the dilatometer, followed by short-time tempering for 100 or $300 \mathrm{~s}$ in the current work. Uncertainties in (b) are less than 0.2 pct (Color figure online).

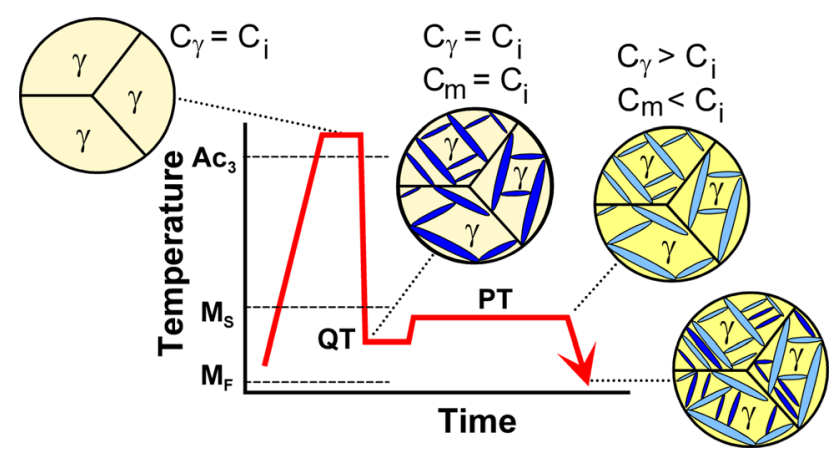

Fig. 17-Schematic of the processing pathway used to create Q\&P (quench and partitioned) advanced high strength steels. Reprinted from Ref. [64]. Note that a 1-step Q\&P heat-treatment is where $\mathrm{QT}=\mathrm{PT}^{[64,66]}$ (Color figure online).

performance, and location-specific microstructures and properties for medium $\mathrm{C}$, low alloy steels produced by Q\&T.

\section{SUMMARY}

From this multiscale, advanced characterization study of Q\&T 4340 steel, with an emphasis on carbide formation during LTT and quench rate effects on microstructural development, the following conclusions can be made:

- Advanced characterization techniques provide new opportunities to study microstructural evolution in steels; a combination of techniques is necessary to deconvolute the subtle changes that occur during Q\&T.

- TEM and HEXRD reveal $\eta$ transition carbide after fast and slow quenching in the as-quenched condition likely associated with autotempering, in addition to after LTT below $300{ }^{\circ} \mathrm{C}$. APT reveals lower than expected $\mathrm{C}$ levels, which could be associated with measurement challenges of fine-scale, carbon-enriched features. Short-time (100 seconds) and conventional tempering (1 hour) at or below $300{ }^{\circ} \mathrm{C}$ results in similar microstructural changes, e.g., transition carbide precipitation.

- The main carbide present after short-time and conventional tempering at or below $300{ }^{\circ} \mathrm{C}$ is $\eta$ transition carbide. No significant element partitioning occurs with respect to the carbide and matrix phases, as determined by APT. The majority of the $\eta$ transition carbide appears to be stoichiometric, as measured by MES, although some non-stoichiometric $\eta$ transition carbide is present. HEXRD also indicates small amounts of cementite are present after quenching, perhaps associated with incomplete austenitization or autotempering of this high $M_{\mathrm{S}}$ temperature steel. Retained austenite decomposition is also thought to occur at higher tempering temperatures (e.g., $200{ }^{\circ} \mathrm{C}$ to $300{ }^{\circ} \mathrm{C}$ ) within the LTT regime. Further study on the role of alloying effects on carbide formation during Q\&T is recommended, in addition to the formation of carbides in austenite relative to the $M_{\mathrm{s}}$ temperature.

- Hägg carbide was found after tempering at $300{ }^{\circ} \mathrm{C}$ or $325^{\circ} \mathrm{C}$, as the fraction of $\eta$ decreases and cementite forms. More work is needed to better 


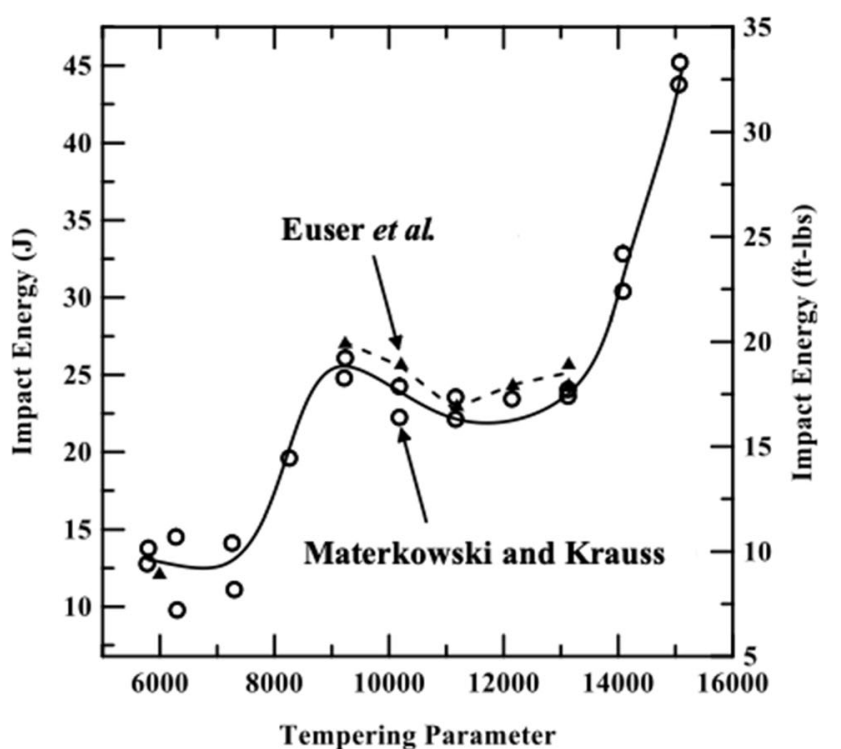

(a)

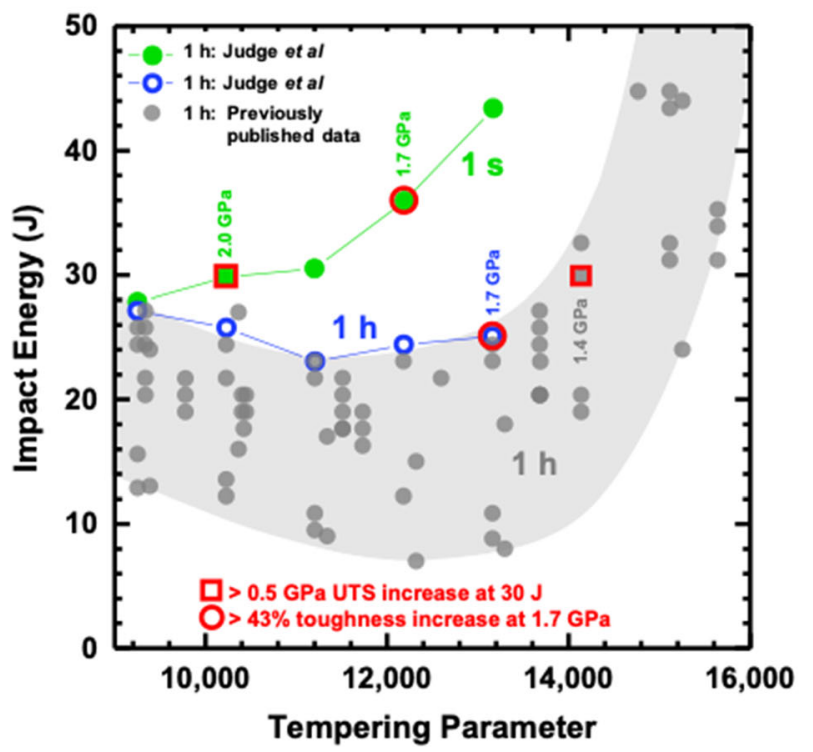

(c)

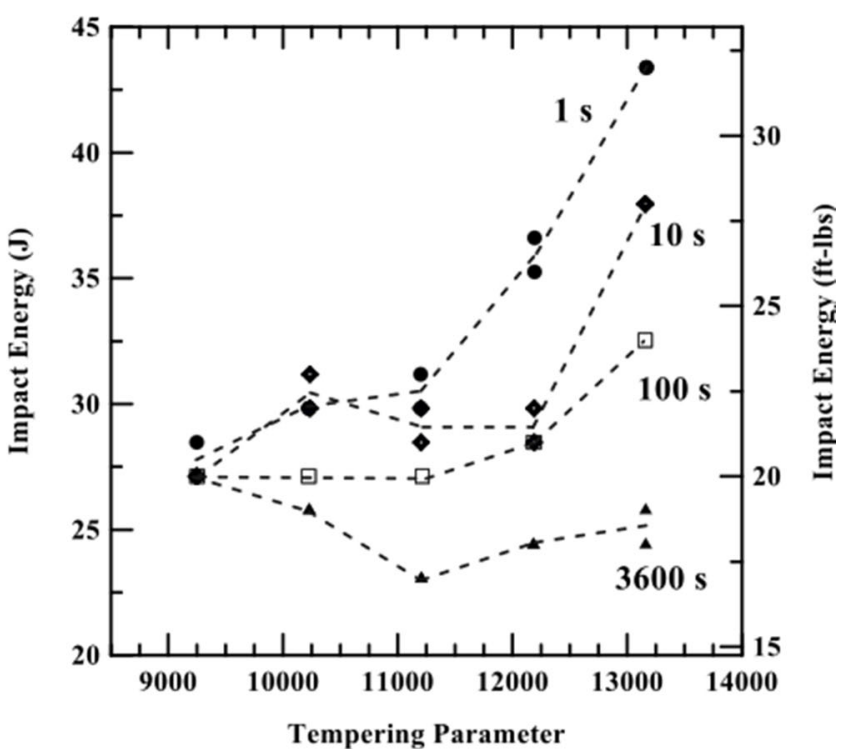

(b)

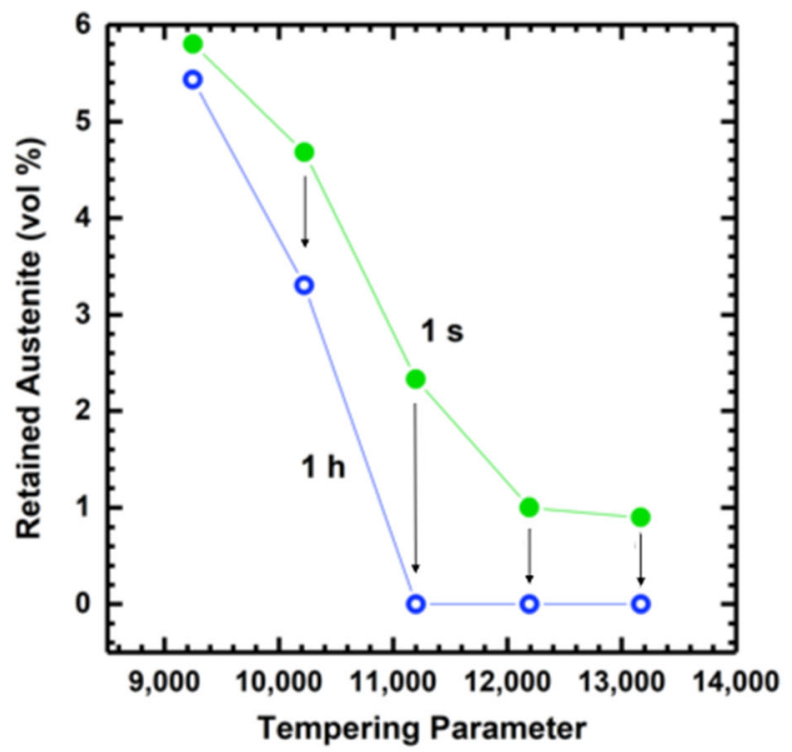

(d)

Fig. 18 - (a) Impact energy vs. the tempering parameter for 4340 from Materkowski and Krauss ${ }^{[8]}$ and after tempering for $3600 \mathrm{~s}$ from Euser et al. Reprinted from Ref. [44]. (b) Impact energy vs. tempering parameter for 4340 after tempering for 1, 10, 100, and $3600 \mathrm{~s}$ and reduced tempered martensite embrittlement with decreasing tempering time. Reprinted from Ref. [44]. (c) A comparison of impact energies produced by short-time and conventional $(1 \mathrm{~h})$ tempering of 4340 ; short-time tempering results in properties not attainable by rapid tempering, ${ }^{[8,45,67-70]}$ and (d) retained austenite vs. tempering parameter for 4340 after $1 \mathrm{~s}$ or $1 \mathrm{~h}$ tempering; greater austenite amounts are retained after short-time tempering. ( $c, d)$ Reprinted from Ref. [45], under the terms of the Creative Commons CC BY license (Color figure online).

understand the transition from $\eta$ transition carbide to cementite and the associated kinetics, and to better determine the time/temperature window in which this occurs.

- Cementite forms after tempering at $325{ }^{\circ} \mathrm{C}$ or above; APT suggests early stage cementite growth under paraequilibrium conditions, followed by initial $\mathrm{Si}$ redistribution and then redistribution of other substitutional alloying elements during late-stage growth $(\mathrm{Cr}, \mathrm{Mn}$, Mo partitions to cementite, particularly near the cementite/matrix interface, and $\mathrm{Si}$ (and $\mathrm{Al}$ and $\mathrm{Ni}^{[14]}$ ) are rejected from the cementite).
- After $575{ }^{\circ} \mathrm{C}$ tempering, coarsened cementite exists (with indications of spheroidization and loss of a well-defined orientation relationship with ferrite/martensite not shown here ${ }^{[14]}$ ) with interfacial $\mathrm{P}$ segregation that may negatively affect fracture properties and delay spheroidization. ${ }^{[14]}$

- Slower quench rates lead to higher fractions of retained austenite in 4340 steel, due to increased C autopartitioning resulting in increased austenite stability, which may be important to subsequent austenite decomposition during Q\&T and creating novel austenite/martensite by Q\&P, for example. 
- Short-time "rapid" tempering results in improved mechanical properties unattainable by conventional tempering, reducing tempered martensite embrittlement (TME), which is likely associated with a decrease in the extent of retained austenite decomposition relative to conventional tempering processes.

- The role of alloying (e.g., Si in 300-M) on microstructural evolution during Q\&T warrants further investigation, and may unlock novel alloying and processing strategies like controlled quenching and/or rapid tempering via induction heating for industrial applications. This new knowledge will also provide further insights into microstructural control (e.g., austenite preservation) and broader applicability of the results presented here to a range of steels subjected to Q\&T and even AHSS processing scenarios like $\mathrm{Q} \& \mathrm{P}$ to produce desirable mechanical property combinations.

\section{ACKNOWLEDGMENTS}

This work was supported by the U.S. Department of Energy through the Los Alamos National Laboratory. Los Alamos National Laboratory is operated by Triad National Security, LLC, for the National Nuclear Security Administration of U.S. Department of Energy (Contract No. 89233218CNA000001). AJC, JKT, and KDC acknowledge support from the Center for Advanced Non-Ferrous Structural Alloys (CANFSA), a National Science Foundation Industry/ University Cooperative Research Center (I/UCRC) [Award No. 1624836], at the Colorado School of Mines and AJC, KDC, JGS, and VKE acknowledge support from the Advanced Steel Processing and Products Research Center (ASPPRC), an Industry/University Cooperative Research Center (I/UCRC), at the Colorado School of Mines during the preparation of this manuscript. This research used resources of the Advanced Photon Source, a U.S. Department of Energy (DOE) Office of Science User Facility operated for the DOE Office of Science by Argonne National Laboratory under Contract No. DE-AC02-06CH11357. APT research was conducted at the Oak Ridge National Laboratory's (ORNL) Center for Nanophase Materials Sciences (CNMS), which is a U.S. DOE Office of Science User Facility. We also gratefully acknowledge the support of M.K. Miller and K.A. Powers from ORNL for their tremendous help and expertise associated with the APT characterization presented in this manuscript.

\section{REFERENCES}

1. G. Krauss: Steel Res. Int., 2017, vol. 88, pp. 1-18.

2. G. Krauss: ISIJ Int., 1995, vol. 35, pp. 349-59.

3. M. Saeglitz and G. Krauss: Metall. Mater. Trans. A, 1997, vol. 28A, pp. $377-87$.
4. G.R. Speich and W.C. Leslie: Metall. Trans., 1972, vol. 3, pp. 1043-54.

5. G. Krauss: Mater. Sci. Eng. A, 1999, vols. 273-275, pp. 40-57.

6. G. Krauss: Steels: Processing, Structure, and Performance, ASM International, Materials Park, 2005, pp. 327-52.

7. D.L. Williamson, R.G. Schupmann, J.P. Materkowski, and G. Krauss: Metall. Trans. A, 1979, vol. 10A, pp. 379-82.

8. J.P. Materkowski and G. Krauss: Metall. Trans. A, 1979, vol. 10A, pp. 1643-51.

9. M. Sarikaya, A.K. Jhingan, and G. Thomas: Metall. Trans. A, 1983, vol. 14A, pp. 1121-33.

10. R.M. Horn and R.O. Ritchie: Metall. Trans. A, 1978, vol. 9A, pp. 1039-53.

11. C.L. Briant: Mater. Sci. Technol., 1989, vol. 5, pp. 138-47.

12. S. Lee, D.Y. Lee, and R.J. Asaro: Metall. Trans. A, 1989, vol. 20A, pp. 1089-1103.

13. F. Zia-Ebrahimi and G. Krauss: Acta Metall., 1983, vol. 32, pp. 1767-77.

14. A.J. Clarke, M.K. Miller, R.D. Field, D.R. Coughlin, P.J. Gibbs, K.D. Clarke, D.J. Alexander, K.A. Powers, P.A. Papin, and G. Krauss: Acta Mater., 2014, vol. 77, pp. 17-27.

15. A. Vieweg, E. Povoden-Karadeniz, G. Ressel, P. Prevedel, T. Wojcik, F. Mendez-Martin, A. Stark, J. Keckes, and E. Kozeschnik: Mater. Des., 2017, vol. 136, pp. 214-22.

16. I. Vieira, J. Klemm-Toole, E. Buchner, D.L. Williamson, K.O. Findley, and E. De Moor: Sci. Rep., 2017, vol. 7, pp. 1-14.

17. Q. Liu, D. Qian, and L. Hua: J. Mater. Sci., 2018, vol. 53, pp. 2774-84.

18. E.J. Mittemeijer, L. Cheng, P.J. Van Der Schaaf, C.M. Brakman, and B.M. Korevaar: Metall. Trans. A, 1988, vol. 19A, pp. 925-32.

19. T. Waterschoot, K. Verbeken, and B.C. De Cooman: ISIJ Int., 2006, vol. 46, pp. $138-46$.

20. Y. Hirotsu and S. Nagakura: Trans. Jpn. Inst. Met., 1974, vol. 15, pp. 129-34.

21. Y. Hirotsu and S. Nagakura: Acta Metall., 1972, vol. 20, pp. $645-55$.

22. S.W. Thompson: Metallogr. Microstruct. Anal., 2016, vol. 5, pp. $367-83$.

23. D.L. Williamson, K. Nakazawa, and G. Krauss: Metall. Trans. A, 1979, vol. 10A, pp. 1351-63.

24. W. Lu, M. Herbig, C.H. Liebscher, L. Morsdorf, R.K.W. Marceau, G. Dehm, and D. Raabe: Acta Mater., 2018, vol. 158 , pp. $297-312$.

25. S.Y.P. Allain, S. Aoued, A. Quintin-Poulon, M. Goune, F. Danoix, J.-C. Hell, M. Bouzat, and M. Soler: Materials, 2018, vol. 11, p. 1087.

26. C.-B. Ma, T. Ando, D.L. Williamson, and G. Krauss: Metall. Trans. A, 1983, vol. 14A, pp. 1033-45.

27. S. Nagakura, Y. Hirotsu, M. Kusunoki, T. Suzuki, and Y. Nakamura: Metall. Trans. A, 1983, vol. 14A, pp. 1025-31.

28. D.H. Sherman, S.M. Cross, S. Kim, F. Grandjean, G.J. Long, and M.K. Miller: Metall. Mater. Trans. A, 2007, vol. 38A, pp. $1698-1711$.

29. S. Ebner, R. Schnitzer, C. Suppan, A. Stark, H. Liu, and C. Hofer: Mater. Charact., 2020, vol. 163, pp. 1-11.

30. B. Kim, C. Celada, D. San Martin, T. Sourmail, and P.E.J. RiveraDiaz-del-Castillo: Acta Mater., 2013, vol. 61, pp. 6983-92.

31. V. Lejay, C. Sidoroff, C. Le Bourlot, M. Perez, and P. Dierickx: Mater. Sci. Technol., 2016, vol. 32, pp. 1106-10.

32. S. Aoued, F. Danoix, S.Y.P. Allain, S. Gaudez, G. Geandier, J.-C. Hell, M. Soler, and M. Goune: Metals, 2020, vol. 10, p. 137.

33. F.G. Caballero, M.K. Miller, A.J. Clarke, and C. Garcia-Mateo: Scr. Mater., 2010, vol. 63, pp. 442-45.

34. F.G. Caballero, M.K. Miller, and C. Garcia-Mateo: Metall. Mater. Trans. A, 2011, vol. 42A, pp. 3660-68.

35. F.G. Caballero, M.K. Miller, C. Garcia-Mateo, C. Capdevila, and S.S. Babu: Acta Mater., 2008, vol. 56, pp. 188-199.

36. C. Zhu, X.Y. Xiong, A. Cerezo, R. Hardwicke, G. Krauss, and G.D.W. Smith: Ultramicroscopy, 2007, vol. 107, pp. 808-12.

37. G. Miyamoto, J.C. Oh, K. Hono, T. Furuhara, and T. Maki: Acta Mater., 2007, vol. 55, pp. 5027-38.

38. Y.X. Wu, W.W. Sun, X. Gao, M.J. Styles, A. Arlazarov, and C.R. Hutchinson: Acta Mater., 2020, vol. 183, pp. 418-37.

39. S.S. Babu, K. Hono, and T. Sakurai: Metall. Mater. Trans. A, 1994, vol. 25A, pp. 499-508. 
40. S.S. Babu, K. Hono, and T. Sakurai: Appl. Surf. Sci., 1993, vol. 67, pp. $321-27$

41. Y. Xiao, W. Li, H.S. Zhao, X.W. Lu, and X.J. Lin: Mater. Charact., 2016, vol. 117, pp. 84-90.

42. C. Zhu, A. Cerezo, and G.D.W. Smith: Ultramicroscopy, 2009, vol. 109 , pp. 545-52.

43. S.J. Barnard, G.D.W Smith, A.J. Garratt-Reed, and J. Vander Sande: in Proc. Int. Conf. Solid-Solid Phase Transform., H.I. Aaronson, D.E. Laughlin, R.F. Sekerka, and C.M. Wayman, eds., Metallurgical Society of AIME, Warrendale, PA, 1982, p. 881.

44. V.K. Euser, D.L. Williamson, K.D. Clarke, K.O. Findley, J.G. Speer, and A.J. Clarke: Metall. Mater. Trans. A, 2019, vol. 50A, pp. 3654-62.

45. V.K. Judge, J.G. Speer, K.D. Clarke, K.O. Findley, and A.J. Clarke: Sci. Rep., 2018, vol. 8, pp. 1-6.

46. M.K. Miller: Atom Probe Tomography, Kluwer Academic/Plenum Press, New York, 2000.

47. F. Vurpillot, A. Bostel, and D. Blavette: Appl. Phys. Lett., 2000, vol. 76 , pp. 3127-29.

48. H.S. Kitaguchi, S. Lozano-Perez, and M.P. Moody: Ultramicroscopy, 2014, vol. 147, pp. 51-60.

49. W. Guo, B.T. Sneed, L. Zhou, W. Tang, M.J. Kramer, D.A. Cullen, and J.D. Poplawsky: Microsc. Microanal., 2016, vol. 22, pp. 1251-60.

50. Y.A. Bagaryatsky: Dokl. Akad. Nauk SSSR, 1950, vol. 73, pp. 1161-64.

51. B.H. Toby and R.B. Von Dreele: J. Appl. Cryst., 2013, vol. 46, pp. $544-49$

52. D.T. Pierce, D.R. Coughlin, D.L. Williamson, K.D. Clarke, A.J. Clarke, J.G. Speer, and E. De Moor: Acta Mater., 2015, vol. 90 , pp. 417-30.

53. D.T. Pierce, D.R. Coughlin, D.L. Williamson, J. Kähkönen, A.J. Clarke, K.D. Clarke, J.G. Speer, and E. De Moor: Scr. Mater., 2016, vol. 121, pp. 5-9.

54. A.J. Clarke, D.R. Coughlin, M.K. Miller, R.D. Field, K.D. Clarke, K.A. Powers, P.J. Gibbs, P.A. Papin, V.K. Judge, D.T. Pierce, D.J. Alexander, E. DeMoor, J.G. Speer, and G. Krauss: Proc. Int. Conf. Solid-Solid Phase Trans. Inorg. Mater., 2015, pp. $1107-14$.

55. K.D. Clarke, A.J. Clarke, R.E. Hackenberg, C.J. Vigil, and C.J. Van Tyne: Proc. Int. Conf. Solid-Solid Phase Trans. Inorg. Mater., 2015, pp. 105-12.
56. K.D. Clarke: in Comprehensive Materials Processing: Thermal Engineering of Steel and Other Alloy Systems, Krauss, G., ed., Elsevier, 2014, vol. 12, pp. 345-61.

57. K.D. Clarke, C.J. Van Tyne, C.J. Vigil, and R.E. Hackenberg: $J$. Mater. Eng. Perform., 2011, vol. 20, pp. 161-68.

58. D.T. Pierce, D.R. Coughlin, K.D. Clarke, E. De Moor, J. Poplawsky, D.L. Williamson, B. Mazumder, J.G. Speer, A. Hood, and A.J. Clarke: Acta. Mater., 2018, vol. 151, pp. 454-69.

59. H. Ino, T. Ito, S. Nasu, and U. Gonser: Acta Metall., 1982, vol. 30, pp. $9-20$

60. J.B. Rellick and C.J. McMahon: Metall. Trans., 1974, vol. 5, pp. 2439-50.

61. E.L. Brown and G. Krauss: Metall. Trans. A., 1986, vol. 17A, pp. 31-36.

62. M. Sarikaya et al., in Proc. of an Int. Conf. on Solid to Solid Phase Transformations, H.I. Aaronson, ed., 1982, pp. 1421-25.

63. D.K. Matlock and J.G. Speer: in Proc. 3rd Int. Conf. Struct. Steels, Lee, H.C., ed., The Korean Institute of Metals and Materials, 2006, pp. 774-81.

64. A. Clarke: PhD Thesis, Colorado School of Mines, 2006.

65. J.G. Speer, E. DeMoor, K.O. Findley, D.K. Matlock, B.C. DeCooman, and D.V. Edmonds: Metall. Trans. A, 2011, vol. $42 \mathrm{~A}$, pp. $3591-3601$.

66. A.M. Streicher, J.G. Speer, D.K. Matlock, B.C. DeCooman: in Proc. of the Int. Conf. on Advanced High-Strength Steels for Automotive Applications, 2004, pp. 51-62.

67. C.H. Shih, B.L. Averbach, and M. Cohen: Trans. ASM, 1956, vol. 48 , pp. $86-118$.

68. C.J. Altstetter, M. Cohen, and B.L. Averbach: Trans. ASM, 1962, vol. 55 , pp. 287-300.

69. C.F. Hickey and A.A. Anctil: J. Heat Treat., 1985, vol. 4, pp. $177-83$.

70. M.G. Hebsur, K.P. Abraham, and Y.V.R.K. Prasad: Eng. Fract. Mech., 1980, vol. 13, pp. 851-64.

71. V.K. Euser, D.L. Williamson, A.J. Clarke, J.G. Speer, ISIJ Int., 2020, in review.

Publisher's Note Springer Nature remains neutral with regard to jurisdictional claims in published maps and institutional affiliations. 